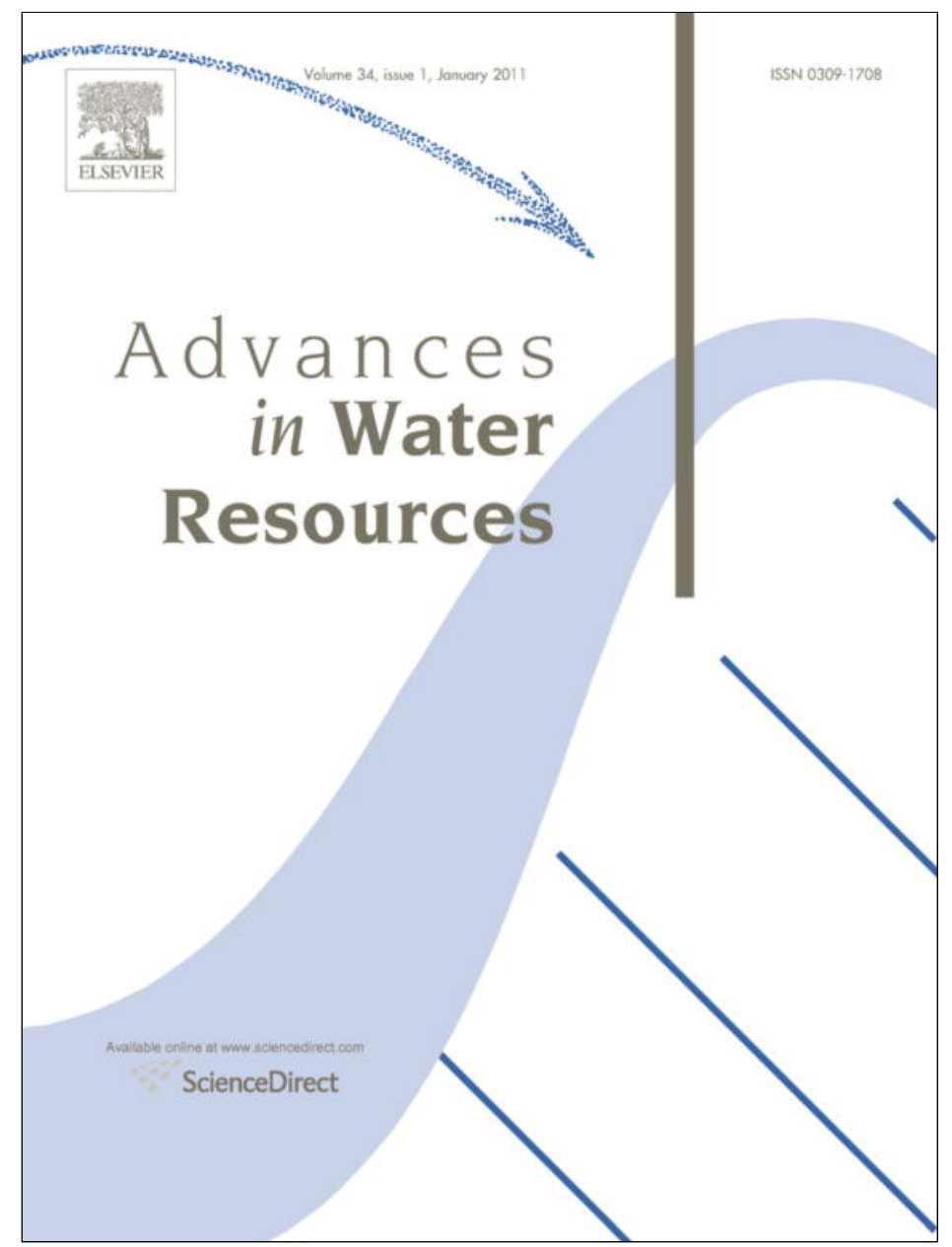

(This is a sample cover image for this issue. The actual cover is not yet available at this time.)

This article appeared in a journal published by Elsevier. The attached copy is furnished to the author for internal non-commercial research and education use, including for instruction at the authors institution and sharing with colleagues.

Other uses, including reproduction and distribution, or selling or licensing copies, or posting to personal, institutional or third party websites are prohibited.

In most cases authors are permitted to post their version of the article (e.g. in Word or Tex form) to their personal website or institutional repository. Authors requiring further information regarding Elsevier's archiving and manuscript policies are encouraged to visit: 


\title{
MAST-2D diffusive model for flood prediction on domains with triangular Delaunay unstructured meshes
}

\author{
C. Aricò ${ }^{\mathrm{a}, *}$, M. Sinagra ${ }^{\mathrm{a}}$, L. Begnudelli ${ }^{\mathrm{b}}$, T. Tucciarelli $^{\mathrm{a}}$ \\ ${ }^{a}$ Dipartimento di Ingegneria Civile, Ambientale ed Aerospaziale, Università di Palermo, Viale delle Scienze Ed. 8, 90128 Palermo, Italy \\ ${ }^{\mathrm{b}}$ Dipartimento di Ingegneria Civile ed Ambientale, Università degli Studi di Trento, Via Mesiano 77, 38123 Trento, Italy
}

\section{A R T I C L E I N F O}

\section{Article history:}

Received 11 April 2011

Received in revised form 8 August 2011

Accepted 12 August 2011

Available online 5 September 2011

\section{Keywords:}

Shallow waters

Finite element method

Diffusive model

Unstructured meshes

Delaunay triangulations

Analytical solution

\begin{abstract}
A B S T R A C T
A new methodology for the solution of the 2D diffusive shallow water equations over Delaunay unstructured triangular meshes is presented. Before developing the new algorithm, the following question is addressed: it is worth developing and using a simplified shallow water model, when well established algorithms for the solution of the complete one do exist?

The governing Partial Differential Equations are discretized using a procedure similar to the linear conforming Finite Element Galerkin scheme, with a different flux formulation and a special flux treatment that requires Delaunay triangulation but entire solution monotonicity. A simple mesh adjustment is suggested, that attains the Delaunay condition for all the triangle sides without changing the original nodes location and also maintains the internal boundaries. The original governing system is solved applying a fractional time step procedure, that solves consecutively a convective prediction system and a diffusive correction system. The non linear components of the problem are concentrated in the prediction step, while the correction step leads to the solution of a linear system of the order of the number of computational cells. A semi-analytical procedure is applied for the solution of the prediction step. The discretized formulation of the governing equations allows to handle also wetting and drying processes without any additional specific treatment. Local energy dissipations, mainly the effect of vertical walls and hydraulic jumps, can be easily included in the model.

Several numerical experiments have been carried out in order to test (1) the stability of the proposed model with regard to the size of the Courant number and to the mesh irregularity, (2) its computational performance, (3) the convergence order by means of mesh refinement. The model results are also compared with the results obtained by a fully dynamic model. Finally, the application to a real field case with a Venturi channel is presented.
\end{abstract}

(c) 2011 Elsevier Ltd. All rights reserved.

\section{Introduction}

The 2D Saint-Venant (SV) [45], or shallow water (SW) equations, are extensively used for hydrodynamic simulations in rivers, lakes, estuaries and floodplains.

Within the framework of the original SV equations, the resulting mathematical model may be classified as dynamic, gravity, diffusion and kinematic wave model, corresponding to different forms of the momentum equation, respectively $[19,58,17]$.

Dynamic wave model retains all the terms of the momentum equation, whereas gravity wave model neglects the effects of bed slope and viscous energy loss and describes flows dominated by inertia. As a matter of fact, the acceleration terms in the SV

\footnotetext{
* Corresponding author.

E-mail addresses: arico@idra.unipa.it (C. Aricò), sinagra@idra.unipa.it (M. Sinagra), lorenzo.begnudelli@unitn.it (L. Begnudelli), tucciar@idra.unipa.it (T. Tucciarelli).
}

equations can be neglected in most practical applications of flood routing in natural channels. The system is thus reduced to a single parabolic equation known as the diffusive wave model. If the water depth gradient term is further omitted, the kinematic wave equation is acquired.

The criteria for demarcating kinematic and diffusive waves have been fully discussed [39-41,47-49,55]. The kinematic model can be easily solved in the case of steep slope or in initially dry areas, where solution of some diffusive models is plagued by instability problems. On the other hand, the kinematic model is not able to compute backwater effects and provides physically inconsistent results when local minima are present in the topographic surface.

The choice of the model to be used for the SW equations solution (fully dynamic, diffusive wave, kinematic wave, 1D or 2D, etc.) depends also on the available input data and on the capability of generating the required hydraulic information in an appropriate format and detail level [50]. These information are, for instance, the topography, the hydraulic properties of the river reaches and 
the inundation zone, the shape of the input flood hydrographs. For example, the solution of the fully dynamic equations is very sensitive to the topographic error, as will be shown in the next section, and simplified models such as the kinematic wave and the uniform formulae do not enable to represent all hydraulic processes during flood events. The diffusive wave used herein is robust with respect to the input data approximations, but provides a higher order accuracy with respect to the kinematic wave and the uniform formulae.

With exception of catastrophic events like dam breaks, flow over inundated plains is often a slow and shallow phenomenon where local free surface slopes are very small. When flood events occur, water is no more contained in the main channel and spills onto the adjacent floodplains. The physical process becomes very complex to simulate and is no longer satisfactorily represented by a 1D scheme. 2D numerical techniques capable of simulating floodplain inundations have been extensively developed in the last years [24].

Numerical techniques, like finite volumes (FV) and finite elements (FE), as well as more conceptually approaches, like storage cell solutions, have been implemented for the solution of these type of problems.

In FV and FE methods the domain is partitioned into cells and the governing equations, written in conservative form, are integrated in each cell. These methods can be applied to both structured and unstructured meshes.

During the last two decades, FV Godunov-type schemes have become popular in seeking the numerical solution of the SW equations. In such schemes a local Riemann problem is solved at every cell interface. Most of these schemes $[2,4,34,54,32]$ have the capability of shock capturing with high accuracy level, but perform well for particular types of flows, for example discontinuous flows over flat topographies and fail in cases of irregular and variable topography or in the appearance of dry areas. Bermudez and Vazquez [14] and Vazquez-Cendon [57] used an upwind discretization of the source term over irregular topography and introduced the concept of C-property: a numerical scheme is regarded as well balanced or satisfying the C-property if it preserves steady-state at rest (stagnant conditions). Upwinding of the source terms is computationally expensive because the source terms have to be projected on a base of the eigenvectors. LeVeque [31] introduced a Riemann problems inside a cell for balancing the source terms and the flux gradients. The method preserve the C-property and the quasi steady-state conditions, but cannot be directly applied to unstructured grids.

Alcrudo and Benkhaldoun [3] used a topography discretization such that a sudden change in the topography occurs at the interface of two cells and solve a Riemann problem at the interface with a sudden change in the bed elevation. This approach leads to several cases of Riemann fan and results are computationally very expensive.

Zhou et al. [61] introduced the surface gradient method, using the water surface elevation to calculate the water depth at cell interfaces. The proposed method maintains the C-property and performs well over variable topography without extra efforts for balancing the source terms and the flux gradients. However the C-property does not hold for unstructured grids.

Several FE approaches have been developed for the SW equations over the past two decades; see, for example, $[27,28$, $33,52,62]$. Much of this effort has involved deriving methods which are stable and non-oscillatory under highly varying flow regimes.

In recent years, FE methods based on discretizing the primitive form of the SW equations using discontinuous approximating spaces have also been studied $[1,2,15,18]$. This discontinuous Galerkin (DG) approach has several appealing features; in particular, the ability to incorporate upwinding and post-processing stability into the solution of highly advective flows. This approach generalizes and extends the Godunov methods: the higher-order polynomials are naturally built into the method and they are defined through the variational equation, instead of computing these higher-order terms by means of ad hoc post processing procedures; diffusive terms are incorporated in the method, while, on the opposite, Godunov schemes do not provide any mechanism for dealing with second-order derivatives.

The DG methods allows for the use of non-conforming grids, that have very useful feature in dealing with complicated geometries. Moreover, the DG method is "locally conservative", that is, the primitive continuity equation relating the change in water elevation to water flux is satisfied in a weak sense element by element. The main drawback of DG methods compared to continuous Galerkin methods is their additional cost. In a DG method, the degrees of freedom of the solution are associated with elements rather than nodal values, and in unstructured Finite Element meshes, there can be substantially more elements than nodes.

One of the main difficulty in the solution of the SW equations is the flow computation over initially dry areas. If no special attention is paid, standard numerical procedure may fail near dry/wet front, producing unphysical oscillations and negative water depths.

During the last 30, 40 years hydrodynamic models have been equipped with Wetting-Drying (WD) algorithms [26]. Maybe the most natural WD approach would be to track the WD interface in time, moving the boundary nodes and deforming accordingly the computational mesh, but a significant computational cost has to be paid for the mesh deformation. For the above mentioned reasons, most of the available WD methods have been developed for fixed mesh. The fixed mesh approach can be divided in two main categories. In the first one, either nodes or entire elements are deactivated when become dry and excluded from the computational domain. However, this inclusion/exclusion of elements may violate the mass and momentum conservation and infringe the numerical stability. In order to describe WD interface that do not match the element interface, some authors [21] introduced transition elements - those where some, but not all nodes, are dry - requiring special treatment. Commonly the transition elements are explicitly detected and their pressure gradient term is neglected. Such discontinuous switches (as cancelling the pressure gradients) make these methods highly non linear and may introduce oscillations and numerical instabilities. Another class of fixed grid WD techniques is the artificial porosity one [21], where the bed is assumed to be porous and non zero water fluxes are allowed for negative depths. The main advantage of this procedure is that the artificial pressure gradient problem is circumvented. For more details about the WD techniques, see $[21,26]$.

Most of the recently proposed floodplains inundation models couple a 1D and a 2D model $[35,16,11,23]$. In Cunge-type storage models, cells correspond to distinct flood compartments and geometric relationships based on water levels are constructed to determine the storage for each flood basin. With the developments in GIS software these relationships are automatically generated from high resolution Digital Elevation Models (DEMs). The abundance of topographic data processed, stored and manipulated within GIS systems has recently led to a fusion of the storage cell concept with raster data format. Such schemes normally use 1D models for main channel flow routing and discretize the floodplains by structured Cartesian (or raster) grid. Each floodplain pixel in the grid is treated as an individual storage cell with inter-cell fluxes treated using uniform flow formulae [11]. The interaction between the main channel and the floodplains is modelled by weir type equations.

Compared to fully explicit Finite Elements, finite differences and finite volumes models, raster-based models have an advantage 
in terms of easy formulation, though questions remain about their simple representation of the flow process [60].

In the present work, a numerical methodology for the solution of diffusive shallow water problem is presented. The governing Partial Differential Equations (PDEs) are discretized over unstructured triangulations using a procedure similar to the linear conforming $P 1$ FE Galerkin scheme but with a different flux formulation.

The methodology follows a fractional time step approach, solving sequentially a prediction and a correction problem. The non linear components of the original PDEs problem are concentrated in the prediction step, while the correction step leads to the solution of a linear system, of the order equal to the number of computational cells. Numerical fluxes discretization is the same in both prediction and correction steps. A proof is given to show that the method is both locally and globally mass conservative.

The prediction step is solved applying the MArching in Space and Time (MAST) methodology, recently proposed for the solution of advection dominated problems [10,5], of the fully dynamic SW equations [6,8], as well as of transport problems in saturated porous media with variable density [7]. MAST peculiarity is to solve at each time step one computational cell after the others according to a given order, such that the mean entering flux is known before the cell solution. This provides an unconditional stability with respect to the time step size, also for Courant (CFL) number much greater than 1 . The requirement for the application of the MAST methodology is the existence of an exact or approximated scalar potential for the flow field. In the present physical problem, an exact scalar potential of the flow field exists and it is the piezometric head. At the beginning of each time step, computational cells are ordered according the their piezometric value. MAST solves a sequence of Ordinary Differential Equations (ODEs), one for each computational cell, from the highest to the lowest potential value.

The present paper is organized as follows: the choice of the diffusive model with respect to the fully dynamic one is first motivated in Section 2 and the governing equations of the diffusive SW equations are presented in Section 3, as well as the applied fractional time step procedure. The spatial discretization of the original governing equation system and the MAST scheme are presented in Section 4, with the numerical flux formulation in a Delaunay triangulation. In Section 5, a simple procedure to obtain a Delaunay mesh from a given set of nodes, also including a subset of fixed edges, is provided. Details of the semi-analytical procedure for the solution of the prediction step are given in Section 6. Handling wetting and drying processes is discussed in the same section. The inclusion of the effect of vertical walls and hydraulic jumps in the proposed model is described in Section 7. In Section 8 , several numerical experiments have been carried out in order to test the efficiency and stability of the proposed model with regard to the size of the CFL number, the computational performance, as well as the convergence order by mesh refinement, which is close to 2 . Numerical results in the case of flow in a rectangular channel are compared with the corresponding ones obtained by other literature models. The flooding from a composite trapezoidal cross section channel in steady-state conditions is studied, as well as the application of a real field case with a Venturi channel. Results of these two last tests are compared with the corresponding ones computed by the fully dynamic model proposed in $[12,13]$.

\section{Why it can be worth using a diffusive model instead of a fully dynamic one?}

Before presenting the new algorithm for the solution of the 2D diffusive shallow water problem (DSW), we provide some most important motivations to prefer the diffusive model instead of the fully dynamic one (FSW). The motivations can be summarized as follows:

(1) The numerical solution of the diffusive model can be computed more quickly, for given mesh size and simulated time, (2) only one boundary condition (b.c.) is required at each boundary point, where the appropriate number of b.c. in the fully dynamic case can be zero, two or three depending on the local Froude number, and (3) most important, the sensitivity of the computed water depth to the topographic error is much higher in the FSW model than in the DSW one.

Motivation (1) is based on the possibility to merge the momentum equations in the continuity equation, in order to get a single higher order equation in only one unknown (instead of three unknowns as for the FSW model) and on the existence of an exact potential. The exact potential and the irrotationality of the flow field allow the application of the MAST procedure, with time steps leading to CFL numbers much larger than one. On the other hand, we have already seen in the introduction that important advances in the solution of the FSW model have recently attained a very good computational efficiency and have made this motivation less compelling than the others.

Motivation (2) is based not only on computational advantages, but also on data limitation. For example, the availability of data regarding supercritical flows entering the upstream domain boundary is often missing and in the FSW solution the normal (i.e. uniform) flow condition is usually adopted to relate water depth to discharge.

Motivation (3) is the most important one. Guinot and Cappallaere [22] have recently analyzed the sensitivity of a FSW 2D model with respect to the parameter errors, where parameters are the topographic elevation, the Manning coefficient and the bed slope. They have shown that, in the very simple case of frictionless, horizontal bed with uniform steady-state flow, the sensitivity can be computed as the solution of a Laplace equation, where the source term is proportional to the quantity:

$a=\left(1-F_{r}^{2}\right)^{-1 / 2}$

where $F_{r}$ is the Froude number. It is well-known that the diffusive model can be thought as a fully dynamic one where the gravity force goes to infinity. This is equivalent to say that the Froude number goes to zero and, in Eq. (1), the quantity $a$ attains its minimum possible size.

The same conclusions can be obtained for the 1D case by observing the behaviour of the water depth when a topographic change is given for constant energy value. See in Fig. 1 the $E(h)$ curve, where $h$ is the water depth and $E$ is the energy per unit weight and constant discharge, relative to the bed level ( $E=h+V^{2} / 2 g$, where $V$ is the mean flow velocity and $g$ the gravity acceleration). The straight line is the potential component of the energy, that is the same water depth $h$.

When a topographic $\Delta z$ change locally occurs, $E$ decreases to $E-\Delta z$. In the diffusive model, $h$ decreases also to $E-\Delta z$ and the piezometric level remains constant. In the fully dynamic model a larger variation $\Delta h$ occurs (see Fig. 1 ), because the water depth reduction has also to balance the velocity and the corresponding kinetic energy increment. If the initial water depth is close to the critical value, the water depth sensitivity in the fully dynamic model approaches infinity, as also suggested by Eq. (1) when the Froude number is close to one.

The sensitivity of the water depth with respect to the topographic error in the complete model strongly overcomes the same sensitivity in the diffusive model only when the Froude number approaches one. On the other hand, the difference between the results of the two models is significant only for the same range of Froude number. If the flow is strongly supercritical, the water 


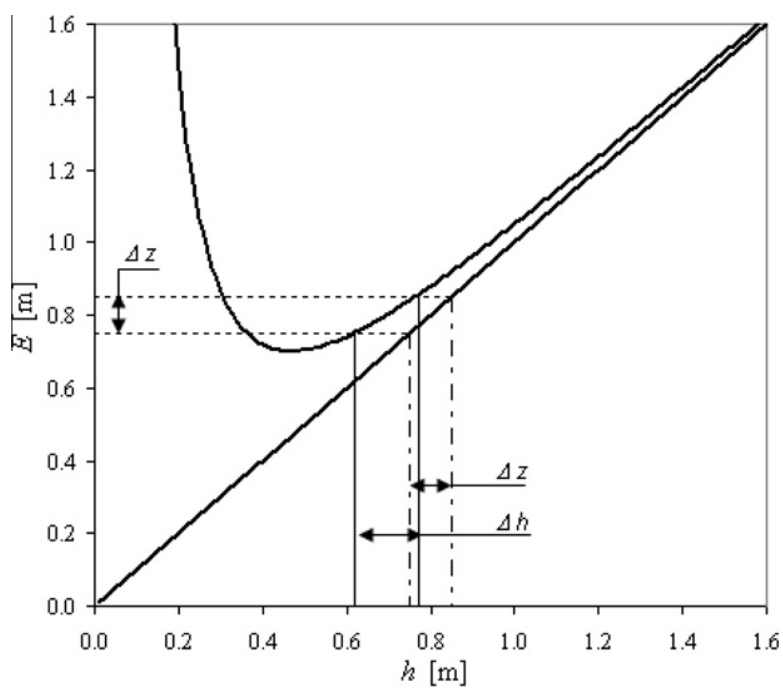

Fig. 1. Variations of water depth in the diffusive model and complete model resulting from local elevation change.

depth is likely to resemble its normal value; if the flow is strongly subcritical, the inertial terms are negligible in the momentum equation.

The choice of the model type still remains subjective and casedependent. In the case of dam-break flows or waves with a length much shorter than the domain extension, inertial terms prevails in the momentum equation and the use of a complete model is compulsory. In all the other cases it is our opinion that diffusive models provide more robust and reliable solutions, as it will be shown to happen in the last two numerical tests, mainly because of the smaller sensitivity to the input data error and uncertainty.

\section{Governing equations system and the fractional time step methodology}

The 2D diffusive form of the shallow water equations can be written as a system of three first order PDEs [56]:

$\frac{\partial H}{\partial t}+\frac{\partial u h}{\partial x}+\frac{\partial v h}{\partial y}=p$

$\nabla_{x} H+\frac{n^{2} u \sqrt{u^{2}+v^{2}}}{h^{4 / 3}}=0$

$\nabla_{y} H+\frac{n^{2} v \sqrt{u^{2}+v^{2}}}{h^{4 / 3}}=0$

where $h$ is the water depth, $H=z+h$ is the piezometric head ( $z$ is the topographic head), $u$ and $v$ are the $x$ and $y$ velocity components, $n$ is the Manning coefficient, $\nabla_{x(y)} H$ is the component of the spatial gradient of the piezometric head in $x(y)$ direction, $p$ represents a source term (e.g., rain intensity). Eq. (2a) is the mass conservation equation and Eqs. (2b) and (2c) are the momentum equations in $x$ and $y$ directions.

Initial and boundary conditions have to be specified to make problem (2) well posed. Boundary conditions may be of Dirichlet (prescribed piezometric head or water depth) or Neumann (prescribed flux) type. If $\Omega$ is the spatial domain where problem (2) is defined, initial and boundary condition can be written as:

$$
\begin{aligned}
& h(\mathbf{x}, t)=h_{D}(\mathbf{x}, t) \quad \text { or } \quad H(\mathbf{x}, t)=H_{D}(\mathbf{x}, t), \quad \mathbf{x} \in \Gamma_{D} \\
& \mathbf{q}(\mathbf{x}, t) \cdot \mathbf{n}=g_{N}(\mathbf{x}, t), \quad \mathbf{x} \in \Gamma_{N} \\
& h(\mathbf{x}, 0)=h_{0} \quad \text { or } \quad H(\mathbf{x}, 0)=H_{0}, \quad \mathbf{x} \in \Omega
\end{aligned}
$$

where $\Gamma=\Gamma_{D} \cup \Gamma_{N}$ is the boundary of $\Omega, \Gamma_{D}$ and $\Gamma_{N}$ are the portions of $\Gamma$ where Dirichlet and Neumann boundary conditions hold respectively, $H_{D}$ and $h_{D}$ are the assigned Dirichlet values for $H$ and $h, g_{N}$ is the assigned Neumann flux, $\mathbf{q}(\mathbf{x}, t)$ is the boundary flow rate vector, $\mathbf{n}$ is the unit outward normal to the boundary, $\mathbf{x}=(x, y)$ and the subscript 0 marks the initial state in the domain.

Eqs. (2b) and (2c) can be merged in Eq. (2a), to get the final one:

$\frac{\partial H}{\partial t}-\frac{\partial}{\partial x}\left(\frac{h^{5 / 3}}{n \sqrt{|\nabla H|}} \frac{\partial H}{\partial x}\right)-\frac{\partial}{\partial y}\left(\frac{h^{5 / 3}}{n \sqrt{|\nabla H|}} \frac{\partial H}{\partial y}\right)=p$

In the proposed procedure, numerical solution of Eq. (4) in the $H$ unknown is attained by means of a time-splitting approach, solving consecutively a prediction and a correction system.

Assume a general system of balance laws:

$\frac{\partial \mathbf{U}}{\partial t}+\nabla \cdot \mathbf{F}(\mathbf{U})=\mathbf{B}(\mathbf{U})$

where $\mathbf{U}$ is the vector of the unknown variables, $\mathbf{F}(\mathbf{U})$ is the flux vector and $\mathbf{B}(\mathbf{U})$ is a source term. Applying a fractional time step procedure, we set:

$\mathbf{F}(\mathbf{U})=\mathbf{F}^{p}(\mathbf{U})+\left(\mathbf{F}(\mathbf{U})-\mathbf{F}^{p}(\mathbf{U})\right)$
$\mathbf{B}(\mathbf{U})=\mathbf{B}^{p}(\mathbf{U})+\left(\mathbf{B}(\mathbf{U})-\mathbf{B}^{p}(\mathbf{U})\right)$

where $\mathbf{F}^{p}(\mathbf{U})$ and $\mathbf{B}^{p}(\mathbf{U})$ are respectively a suitable numerical flux and source term, further defined. After integration in time, system (5) can be split in the two following ones:

$\mathbf{U}^{k+1 / 2}-\mathbf{U}^{k}+\nabla \cdot \int_{0}^{\Delta t} \mathbf{F}^{p} d t=\int_{0}^{\Delta t} \mathbf{B}^{p} d t$

$\mathbf{U}^{k+1}-\mathbf{U}^{k+1 / 2}+\nabla \cdot \int_{0}^{\Delta t} \mathbf{F} d t-\nabla \cdot \overline{\mathbf{F}}^{p} \Delta t=\int_{0}^{\Delta t} \mathbf{B} d t-\overline{\mathbf{B}}^{p} \Delta t$

where $\overline{\mathbf{F}}^{p}$ and $\overline{\mathbf{B}}^{p}$ are the mean numerical flux and source terms computed along the prediction step, $\mathbf{U}^{k+1 / 2}$ and $\mathbf{U}^{k+1}$ are the unknown variables computed respectively at the end of the prediction and the correction phases. Integrals $\overline{\mathbf{F}}^{p} \Delta t$ and $\overline{\mathbf{B}}^{p} \Delta t$ will be estimated "a posteriori" after the solution of the prediction problem, according to the procedure explained in the next section. We call systems (7a) and (7b) prediction and correction systems respectively. Observe that summing systems (7a) and (7b), the integral of the original system (5) is formally obtained. The difference between $\mathbf{U}^{k+1}$ and $\mathbf{U}^{k+1 / 2}$ in Eq. (7b) is close to zero as far as the difference between the predicted and mean in time values of the fluxes and source terms is small. The advantage of using formulations (7) instead of (5) is that, with a suitable choice of the prediction terms $\mathbf{F}^{p}(\mathbf{U})$ and $\mathbf{B}^{p}(\mathbf{U})$, each of the two systems (7a) and (7b) can be much easier to solve than the original system (5).

In the present case we have:

$\mathbf{U}=H$

$\mathbf{F}=-\frac{h^{5 / 3}}{n \sqrt{|\nabla H|}} \nabla H$

$\mathbf{B}=p$

We set:

$\mathbf{F}^{p}=-\frac{h^{5 / 3}}{n \sqrt{|\nabla H|^{k}}}(\nabla H)^{k}$

$\mathbf{B}^{p}=\mathbf{B}$

where index $k$ marks the beginning of the time step (time level $t^{k}$ ). Observe that the flux formulation of the prediction step differs from the original one (Eq. (4)) in the time level of the gradients of $H$. In the prediction step, spatial gradients of the piezometric head are assumed constant in time and equal to the values computed at the end of the previous time step. The prediction equation to be solved along the given time step is: 
$\frac{\partial H}{\partial t}-\frac{\partial}{\partial x}\left(\frac{h^{5 / 3}}{n \sqrt{\left|\nabla H^{k}\right|}} \frac{\partial H^{k}}{\partial x}\right)-\frac{\partial}{\partial y}\left(\frac{h^{5 / 3}}{n \sqrt{\left|\nabla H^{k}\right|}} \frac{\partial H^{k}}{\partial y}\right)=p$

The prediction problem is solved in its integral form, as shown in the following, while the correction problem is solved in its differential linearized form:

$\frac{\partial \eta}{\partial t}-\frac{\partial}{\partial x}\left(\frac{\left(h^{k m}\right)^{5 / 3}}{n \sqrt{\left|\nabla H^{k}\right|}} \frac{\partial(\eta-\vartheta)}{\partial x}\right)-\frac{\partial}{\partial y}\left(\frac{\left(h^{k m}\right)^{5 / 3}}{n \sqrt{\left|\nabla H^{k}\right|}} \frac{\partial(\eta-\vartheta)}{\partial y}\right)=0$

where

$\eta=H-H^{k+1 / 2}$

$h^{k m}=\frac{h^{k}+h^{k+1 / 2}}{2}$

$\vartheta=H^{k}-H^{k+1 / 2}$

with initial condition $\eta=0$. Index $k+1 / 2$ marks the solution of the prediction Eq. (10).

After some simple manipulations, it can be shown that the quasi-linear differential form of the prediction problem is kinematic, with only one characteristic line passing through each $(\mathbf{x}, t)$ point. The prediction PDE system is equivalent to a single non-linear convection equation, function of the gradient of the piezometric head at time level $t^{k}$, while correction system has the functional characteristics of a pure diffusive process. For these reasons we call the prediction and the correction systems respectively convective prediction system and diffusive correction system.

The convective prediction problem has to be solved by giving the known discharge as boundary condition to the upstream nodes. The diffusive correction system is solved by setting to zero diffusive flux in the upstream boundary nodes and by giving to the downstream nodes the proper boundary condition required to satisfy the boundary conditions of the original problem (2). For example, if the downstream water level is known and equal to $H^{*}$, the correction $\eta$ in the downstream boundary will be set equal to: $\eta=H^{*}-H^{k+1 / 2}$

\section{The MAST procedure}

The spatial discretization of the governing equations is carried out on a generally unstructured triangular mesh that satisfies the Delaunay property. A Delaunay triangulation in $\mathbb{R}^{2}$ is defined by the condition that all the nodes in the mesh are not interior to the circles defined by the three nodes of each triangle. Let $\Omega \subset \mathfrak{R}^{2}$ be a bounded domain, $\Omega_{h}$ a polygonal approximation of $\Omega$ and $T_{h}$ an unstructured Delaunay-type triangulation of $\Omega_{h}$. The triangulation $T_{h}$ is called basic mesh and the triangle $k_{T} \in T_{h}$ is called primary element. Let $P_{h}=\left\{P_{i}, i=1, \ldots, N\right\}$ be the set of all vertices (nodes) of all $k_{T} \in T_{h}$ and $N$ the total number of nodes. The dual mesh $E_{h}=\left\{e_{i}, i=1, \ldots, N\right\}$ is constructed over the basic mesh. The dual finite volume $e_{i}$ associated with the vertex $P_{i}$ is the closed polygon given by the union of sub-triangles resulting from the subdivision of each triangle of $T_{h}$ connected to node $P_{i}$ by means of its axes (see Fig. 2). In the following of the paper the dual volumes $e$ are called also cells. The sub-triangles are called secondary elements and are indicated as $e^{I I}$. Cells $e_{i}$ satisfy:

$\Omega=\cup e_{i}$

The dual finite volume of the Delaunay triangulation, previously defined, is called Voronoi cell or Thiessen polygon [42]. Each Voronoi cell $e_{i}$ associated to node $P_{i}$ consists of the points $Q$ such that $d\left(Q, P_{i}\right) \leqslant d\left(Q, P_{j}\right)$ for $j=1, \ldots, N$ and $j \neq i\left(d\left(Q, P_{i}\right)\right.$ is the distance

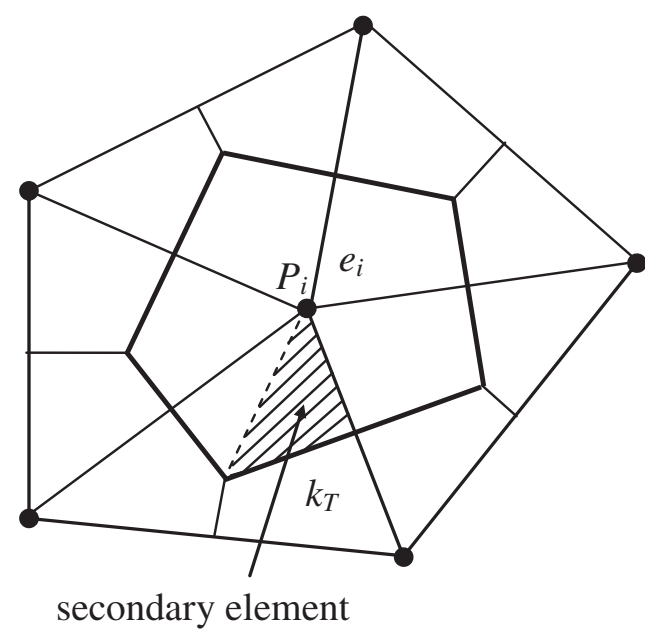

Fig. 2. The basic mesh and the dual finite volume mesh.

between $Q$ and $P_{i}$ ). The vertices of the Voronoi cells are the circumcentres of the Delaunay triangulation.

The storage capacity is assumed concentrated in the cells (nodes) in the measure of $1 / 3$ of the area of all the triangles sharing each node. A linear variation of the piezometric head $H$ inside each triangle of the mesh is assumed on the base of the values at its three nodes. After integration in space, the differential form of the prediction system (10) is:

$A_{i} \frac{d H_{i}}{d t}+\sum_{j} F l_{i, j}^{\text {out }}=\sum_{m} F l_{i, m}^{\text {in }}+A_{i} p_{i}, \quad i=1, \ldots, N$

with

$F l_{i, j}^{\text {out }}=K_{i, j}^{k} h_{i}^{5 / 3}, \quad A_{i}=\frac{1}{3} \sum_{n=1, N_{T}}\left|k_{T}\right|_{n} \delta_{i, n}$

where $A_{i}$ is the area of cell $i, N_{T}$ is the total number of triangles, $\left|k_{T}\right|_{n}$ is the area of triangle $n, \delta_{i, n}$ is the Kronecker delta equal to one or zero according if node $i$ is or is not a vertex of triangle $n, F l_{i, j}^{\text {out }}$ is the flux going from cell $i$ to the any neighbouring downstream (in the potential scale) cell $j$ with $H_{j}^{k} \leqslant H_{i}^{k}$, flux coefficient $K_{i, j}^{k}$ will be further defined, $F l_{i, m}^{i n}$ is the flux entering in cell $i$ from any neighbouring upstream cell $m$ with $H_{i}^{k} \leqslant H_{m}^{k}$ and $p_{i}$ is source term in node $i$.

Solution of system (14a) can be disentangled in the sequential solution of $N$ equations by approximating the r.h.s. with its mean value along the given time step, that is by setting:

$A_{i} \frac{d H_{i}}{d t}+\sum_{j} F l_{i, j}^{\text {out }}=\sum_{m} \overline{F l_{i, m}^{i n}}+A_{i} \overline{p_{i}}$

where $\overline{F l} l_{i, m}^{i n}$ is the mean in time value of the flux entering from cell $m$, previously solved, and $\overline{p_{i}}$ is the $p_{i}$ mean value.

At each time step, the computational cells are ordered according to the decreasing value of their potential (the piezometric head), computed at the end of the previous time step and then are sequentially solved throughout the computational domain.

After solution of each ODE (15), the mean in time total flux going from cell $i$ to the neighbouring downstream cells can be computed by the local mass balance for cell $i$, that is

$\overline{F l}_{i}^{\text {out }}=\overline{F l_{i}^{i n}}-A_{i} \frac{h_{i}^{k+1 / 2}-h_{i}^{k}}{\Delta t}+A_{i} \overline{p_{i}}$

where $\overline{F l_{i}^{\text {out }}}$ and $\overline{F l_{i}^{i n}}$ are respectively the total mean leaving and entering fluxes, with 
$\overline{F l_{i}^{i n}}=\sum_{m} \overline{F l_{i, m}^{i n}}$

and $h_{i}^{k+1 / 2}$ is the final value of the water depth computed by the prediction step.

The mean flux $\overline{F_{i, j}}$ out going from cell $i$ to cell $j$ with $H_{i}^{k}>H_{j}^{k}$ can be estimated by partitioning $\overline{F l}_{i}^{\text {out }}$ according to the ratio between the flux $F_{i, j}^{\text {out }}$ and the sum of the leaving fluxes at the end of the prediction step, that is:

$\overline{F l_{i, j}^{\text {out }}}=\overline{F_{i}^{\text {out }}} \frac{\left(F l_{i, j}^{\text {out }}\right)^{k+1 / 2}}{\sum_{l}\left(F l_{i, l}^{\text {out }}\right)^{k+1 / 2}}=\overline{F l_{i}^{\text {out }}} \frac{K_{i, j}^{k}}{\sum_{l} K_{i, l}^{k}}$

where the sum is extended to all the neighbouring cells $l$ with $H_{l}^{k} \leqslant H_{i}^{k}$. Finally, the mean in time fluxes entering in cells $j$ with lower total head can be computed as:

$\overline{F l}_{i, j}^{\text {out }}=\overline{F l}_{j, i}^{\text {in }}$

After solution of the ODE corresponding to cell $i$, the next equation to be solved is relative to the cell $j$ with the maximum piezometric head among the unsolved ones and $H_{j}^{k} \leqslant H_{i}^{k}$. Observe that, because of the chosen equations sorting, the mean entering fluxes will be always known before each ODE solution.

Eq. (16) represents the local mass continuity equation integrated in space and time inside the Voronoi cell and its application guarantees the global conservation of the mass (see also Appendix A). The solution of the prediction problem can be classified as "explicit", because it depends only on the initial state in the cell and on the information (i.e. the flux) coming from the upstream (in the potential scale) cells, previously solved.

Differently from the previous MAST formulations ([6,8]), we compute the flux coefficient $K_{i, j}^{k}$ (see Eq. (14b)) as:

$K_{i, j}^{k}=\left(c_{i, j}^{1} E_{1}^{k}+c_{i, j}^{2} E_{2}^{k}\right) \frac{H_{i}^{k}-H_{j}^{k}}{d_{i j}}$

with

$c_{i, j}^{1}=d_{i, j}^{1}, \quad c_{i, j}^{2}=d_{i, j}^{2} \quad$ if $d_{i, j}^{1} \geqslant 0$ and $d_{i, j}^{2} \geqslant 0$

$c_{i, j}^{1}=d_{i, j}^{1}+d_{i, j}^{2}, \quad c_{i, j}^{2}=0 \quad$ if $d_{i, j}^{1} \geqslant d_{i, j}^{2}$ and $d_{i, j}^{2}<0$

$c_{i, j}^{1}=0, \quad c_{i, j}^{2}=d_{i, j}^{1}+d_{i, j}^{2} \quad$ if $d_{i, j}^{2} \geqslant d_{i, j}^{1}$ and $d_{i, j}^{1}<0$

where $d_{i j}$ is the distance between nodes $i$ and $j$ and $d_{i j}^{m}$ is the distance between the circumcentre of each element $m=1,2$ sharing edge $i j$ from the same edge, that is:

$d_{i, j}^{m}=\frac{\left(x_{m}-x_{12}\right)\left(y_{j}-y_{i}\right)-\left(y_{m}-y_{12}\right)\left(x_{j}-x_{i}\right)}{\sqrt{\left(x_{j}-x_{i}\right)^{2}+\left(y_{j}-y_{i}\right)^{2}}} \delta(m)$

where $x_{m}, y_{m}$ are the circumcentre coordinates, $x_{12}$ and $y_{12}$ are the coordinates of the common edge midpoint (point $P_{12}$ in Fig. 3)

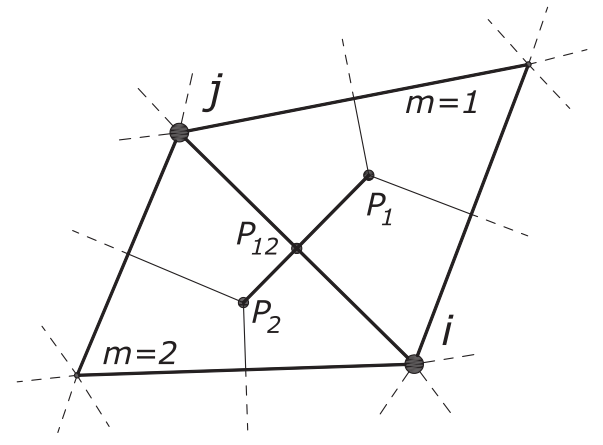

Fig. 3. Circumcentres $P_{1}$ and $P_{2}$ of elements 1 and 2 sharing edge $i j$. and $\delta(m)=-1$ or 1 if direction $i j$ is respectively counterclockwise or not in triangular element $m$. Observe that $d_{i, j}^{m}<0$ if the angle opposite to side $i j$ in triangle $m$ is obtuse. If the edge $i j$ belongs to the external boundary of the domain we set:

$K_{i, j}^{k}=d_{i, j}^{1} E_{1}^{k} \frac{H_{i}^{k}-H_{j}^{k}}{d_{i j}}$

and we assume that $d_{i, j}^{1} \geqslant 0$ (as better explained in the next section). Coefficient $E_{m}^{k}$ is equal to:

$E_{m}^{k}=\frac{1}{n_{m} \sqrt{\left|\Delta H_{m}^{k}\right|}}$

where sub-index $m$ marks all parameters of triangular element $m$.

The new adopted space discretization is similar to the one of the standard linear conforming Galerkin Finite Element scheme. According to Eq. (14b) and Eqs. (19a)-(19e), the flux $\mathrm{Fl}_{i, j}^{\text {out } m}$ moving from cell $i$ to cell $j$ in each of the two triangles $m$ sharing side $i j$, is computed as:

$F_{i, j}^{o u t, m}=c_{i, j}^{m} E_{m}^{k} \frac{H_{i}^{k}-H_{j}^{k}}{d_{i j}} h_{i}^{5 / 3}$

It can be shown that in the Galerkin formulation the same flux is computed as $[42,43]$ :

$F l_{i, j}^{\text {out }, m}=d_{i, j}^{m} E_{m}^{k} \frac{H_{i}^{k}-H_{j}^{k}}{d_{i j}} \hat{h}_{m}^{5 / 3}$

where $\hat{h}_{m}$ is the average in space water depth inside triangle $m$. In both cases Eqs. (22a) and (22b) approximate the flux between cells $i$ and $j$, through side $d_{i, j}^{m}$ of the Voronoi polygons of cell $i$ and cell $j$, due to the piezometric head difference $H_{i}^{k}-H_{j}^{k}$. The difference of the MAST inter-cell flux formulation with respect to the Galerkin scheme, motivated and discussed in the next section for the case of Delaunay unstructured meshes, does not affect the flux computation between two cells with constant water depth and sharing parts of two acute triangles.

Diffusive problem (11) and (12) is solved using the same spatial discretization adopted in the prediction problem, as well as a fully implicit time discretization. Integration of Eq. (11) inside each Voronoi cell leads to the following system:

$\frac{A_{i}}{\Delta t} \eta_{i}+\sum_{n=1, N_{T}} D_{i, j}^{k}\left(\eta_{i}-\eta_{j}\right) \delta_{i, n}=\sum_{n=1, N_{T}} D_{i, j}^{k}\left(\vartheta_{j}-\vartheta_{i}\right) \delta_{i, n}, \quad i=1, \ldots, N$

with

$D_{i, j}^{k}=\sum_{m=1,2} \frac{c_{i, j}^{m} E_{m}^{k}}{d_{i j}}\left(h_{l}^{k m}\right)^{5 / 3}$

where $\delta_{i, n}$ is the Kronecker delta, equal to one or zero according if node $i$ is or is not a vertex of triangle $n$ and the sum in Eq. (23b) is extended to the two triangles $m$ sharing side $i j . \quad l=i$ if $H_{i}^{k} \geqslant H_{j}^{k}, l=j$ if $H_{i}^{k}<H_{j}^{k}$. Differently from the previous formulations of the diffusion coefficients $D_{i, j}^{k}$, the new one provides the same flux estimation of the prediction problem, for given element parameter $E_{m}^{k}$ and node variable difference, that is $H_{i}^{k}-H_{j}^{k}$ in the prediction problem and $\eta_{i}-\eta_{j}$ in the correction problem.

Fully implicit time discretization provides unconditional stability, along with some approximation error in the solution [30]. The approximation error is small because its magnitude is of the same order of the computed correction $\eta$, and the source term on the rhs of Eq. (23a) goes to zero along with the time step size. Even during abrupt potential changes, the potential correction will be small with respect to the predicted change. This implies that the absolute error in the estimation of the piezometric correction will only 
weakly affect the piezometric final value computed at time level $k+1$.

The linear system resulting from Eqs. (23) has order equal to the number of the nodes and is well conditioned, with a matrix that is always symmetric, positive definite and, according to the new flux formulation, strictly diagonally dominant, even in the case of Delaunay triangulations with obtuse triangles (see next section). It can be shown (see Appendix B) that this last property guarantees the steady-state monotonicity of the solution, that remains regular also preserving the local mass conservation, even in the parts of the domain with sharp topographic changes, like along the banks of a trapezoidal channel.

After solution of the linear system (23) is obtained in the $\eta$ unknowns, the piezometric heads $H$ at the end of the time step are obtained as:

$H^{k+1}=H^{k+1 / 2}+\eta$

A major property of the MAST scheme, as herein formulated, is that in steady-state condition the correction component vanishes for any arbitrary chosen time step size. This relevant result is due to the use of the same spatial discretization for the computation of both the convective and the diffusive fluxes.

The proposed scheme can be regarded as a finite volume (FV) method where the control volume is the Voronoi cell, similar to the standard conforming Galerkin Finite Element scheme. MAST scheme is a locally conservative method, meaning that the sum of the fluxes over each cell edges equals the accumulation term plus any sources or sinks in the cell, and the flux is continuous across each edge [29] (see Appendix C).

\section{The required generalized Delaunay property}

If one edge $i j$ linking nodes $i$ and $j$ is opposite to the obtuse angle of an element $m$, the distance $d_{i, j}^{m}$ of the circumcentre from edge $i j$, defined in Eq. (19e), is negative and three possibilities exist.

The first possibility is that edge $i j$ is common to two elements and these have the sum of their distances $s_{i j}=d_{i, j}^{1}+d_{i, j}^{2}$ greater than or equal to zero, such that the circumcentre $P_{1}$ of the obtuse triangle is located on the axis of the edge $i j$ between the same edge and the circumcentre $P_{2}$ (Fig. 4a). It can be shown $[20,42]$ that this condition is equivalent to have the third node of the first (or second) triangle out of the circle defined by the three nodes of the second (or first) triangle. This implies that the Delaunay property guarantees the condition (see Fig. 4b):

$d_{i, j}^{1}+d_{i, j}^{2} \geqslant 0$

for all the edges of the mesh shared by two triangles. Most of the today available mesh-generators satisfy the Delaunay property, even if some exceptions occur around internal boundaries, or when the mesh density is forced to change in given subdomains. If the

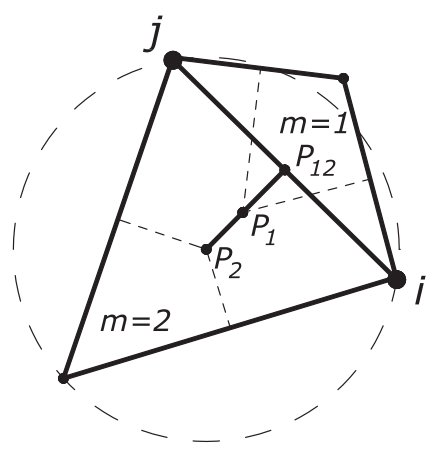

Fig. 4a. Elements 1 and 2 satisfy the Delaunay property.

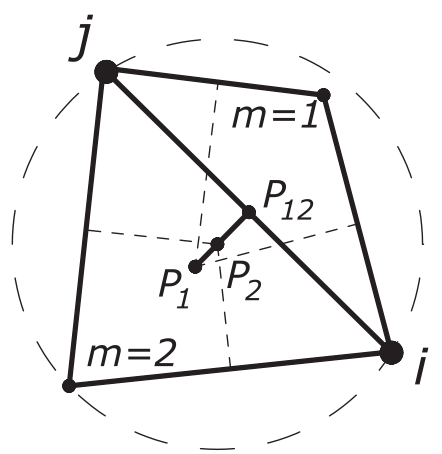

Fig. 4b. Elements 1 and 2 do not satisfy the Delaunay property.

Delaunay property is satisfied, both the inter-cell fluxes computed by Eq. (22a) in the two elements sharing edge $i j$ are either oriented according to the difference between the water levels of the two cells or zero. Observe that in the standard Galerkin Finite Element discretization, if the two element fluxes on the r.h.s. of Eq. (2c) are computed with different parameters $E_{m}^{k}$, the sign of the total flux from node $i$ to node $j$ can loose consistency with the piezometric difference even if the mesh satisfies the Delaunay property and the sum of the distances $s_{i j}=d_{i, j}^{1}+d_{i, j}^{2}$ is positive.

The second possibility (Fig. $4 \mathrm{~b}$ ) is that the Delaunay property is not satisfied. In this case it is still possible to obtain a new mesh that satisfies condition (25a) for all the internal edges, starting from the original one, without changing the original node location. This can be done by a series of local edge swaps, where two elements sharing the same edge are changed in a new couple, sharing the same nodes but a different edge, connecting the two nodes opposite to the previous edge. See for example the new triangles obtained in Fig. $5 b$ by the original ones of Fig. 5a. It can be shown [20] that the common edge satisfies the Delaunay property in at least one of the two configurations. By iterating the same control for all the edges, the Delaunay property is quickly attained for all the edges of the mesh that are shared by two triangles.

The third possibility is that the element $m$ is a boundary element and $i j$ is a boundary edge opposite to an obtuse angle. In this case the flux coefficient $K_{i, j}^{k}$ in Eq. (20) remains negative, even if the mesh satisfies the Delaunay property, because the distance of the circumcentre from the boundary edge is negative. We define Generalized Delaunay mesh a Delaunay mesh where the condition:

$d_{i, j}^{1} \geqslant 0$

holds for all the boundary edges.

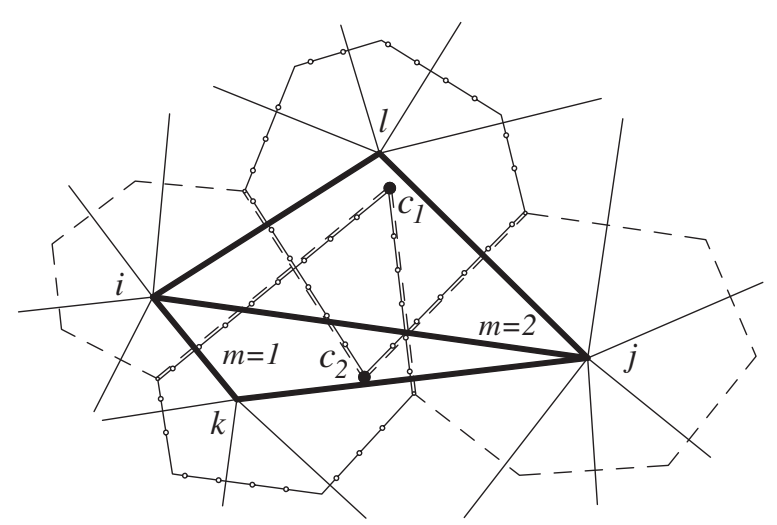

Fig. 5a. Original not Delaunay triangulation. 


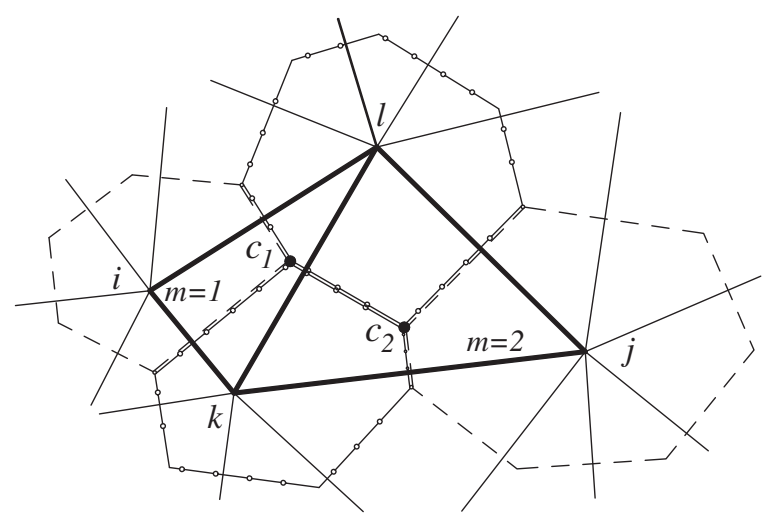

Fig. 5b. Delaunay triangulation after the mesh correction (edge swap).

If condition (25b) does not hold for one or more boundary edges, and/or common edges are fixed as internal boundaries, it is still possible to obtain a Generalized Delaunay mesh, also saving the internal boundaries. To this aim the two original triangles sharing the internal boundary or the original triangle with a boundary edge opposite to an obtuse angle are divided in new triangles by adding new nodes along the original edge. The new triangles have the same height as the original ones with respect to the boundary edge, but the base length will be a fraction of the original one. After this change, the same edge swap iterative procedure can be applied to the resulting edges, with no exception for the new ones located on the internal boundary. It can be easily shown (see Appendix D) that the resulting mesh will satisfy the Generalized Delaunay property, if the number of new nodes is large enough. See an example in Figs. $6 a$ and $6 b$ where the boundary edges $2-3$ and $1-4$ do not satisfy the Generalized Delaunay property (Fig. 6a). The mesh is first changed in a new mesh by setting a new node in both edges 2-3 and 1-4 (Fig. 6b), and then changed in a Generalized Delaunay mesh by applying the swap technique to the edge $4-5$, that is changed with the new edge 3-7 (Fig. 6c).

The same spatial discretization adopted for the convective fluxes is also applied for the estimation of the diffusive fluxes. In

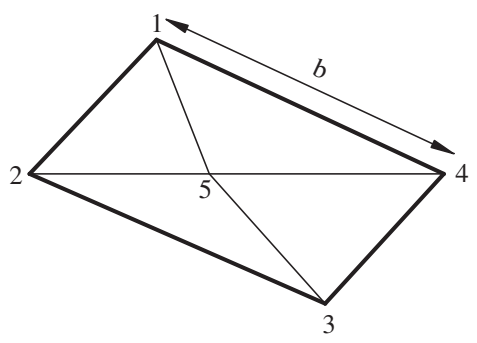

Fig. 6a. Original triangulation

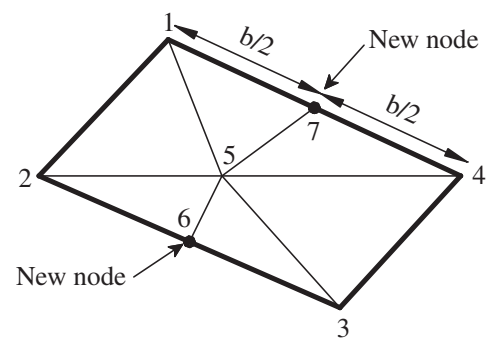

Fig. 6b. New triangulation after adding two new nodes.

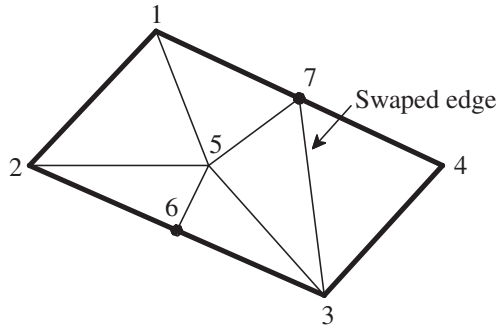

Fig. 6c. Generalized Delaunay triangulation after applying the swap technique.

Appendix $\mathrm{E}$ it is shown that this implies for the resulting linear system matrix the so called M-property [59], that is the negativity of all the extra-diagonal matrix coefficients. The M-property guarantees inter-cell fluxes with a sign that is always consistent with the sign of the corresponding water level difference. An important consequence is the monotonicity of the steady-state solution, when source terms are missing, as well as the lack of spatial oscillations [59].

Observe that the diffusive fluxes computed with the spatial discretization of Eq. (11) according to the standard Galerkin approach, are proportional to a parameter $T$ assumed constant inside each element $m$ and equal to $[42,43]$ :

$$
T^{m, k}=E_{m}^{k} \hat{h}_{m}^{5 / 3}=\frac{\hat{h}_{m}^{5 / 3}}{n_{m} \sqrt{\left|\Delta H_{m}^{k}\right|}}
$$

The Galerkin approach guarantees the positive definite condition (all the eigenvalues greater than zero) of the final linear system matrix, even if the Generalized Delaunay condition does not hold, but does not guarantee the M-property [30,42]. On the other hand, if a non Generalized Delaunay mesh is used with the proposed algorithm, the iterative methods used for the solution of the linear system in the correction problem can fail, because of the negative eigenvalues. This restricts the use of the proposed algorithm to triangular meshes that satisfy conditions (25a) or (25b) in all the edges.

Solving the convective and the diffusive problem using the same computational cells and flux spatial discretization is very important, because the use of different formulation for the computation of convective and diffusive fluxes can lead to small oscillations in space and in time even in the case of steady-state flow, when the dissipative correction is expected to go to zero in the MAST procedure. In the proposed algorithm, the fluxes computed by Eq. (26a) are proportional to a parameter $T$ given by:

$T_{i, j}^{m, k}=E_{m}^{k} h_{i}^{5 / 3}=\frac{h_{i}^{5 / 3}}{n_{m} \sqrt{\left|\Delta H_{m}^{k}\right|}}$

that cannot be thought as an element parameter. This implies that the velocity is not constant inside the element and divergence is not zero in each point of the domain, even if local and global mass conservation are both satisfied in the spatially discretized domain.

\section{A semi-analytical solution of the prediction problem}

In the previous formulation of the MAST algorithm the solution of each ODE problem (15) has been sought after numerically, adopting a Runge-Kutta scheme with a self adapting time step, a fraction of the original one [6]. In the present formulation, an approximated analytical solution is provided for the solution of the problem (15). Call $h_{i}^{k}$ the water depth at the beginning of the 
time step and $h_{i}^{k f}$ its asymptotic steady-state value (i.e. when $d H_{i}$ / $d t=0$ ), computed according to Eqs. (15) and (17), that is:

$h_{i}^{k f}=\left(\frac{\overline{F l}_{i}^{i n}}{\sum_{j} K_{i, j}^{k}}\right)^{3 / 5}$

with

$\overline{F l_{i}^{i n}}=\overline{F l_{i}^{i n}}+A_{i} \overline{p_{i}}$

Eq. (15) can be written in dimensionless form as:

$\frac{d \xi}{d \tau}=1-\xi^{5 / 3}, \quad \xi=\frac{h_{i}}{h_{i}^{k f}}, \quad \tau=\frac{d t \bar{F} l_{i}^{i n}}{A_{i} h_{i}^{k f}} \quad$ if $h_{i}^{k f}>h_{i}^{k}$

$\frac{d \xi}{d \tau}=\xi_{f}^{5 / 3}-\xi^{5 / 3}, \quad \xi=\frac{h_{i}}{h_{i}^{k}}, \quad \tau=\frac{d t \overline{F l_{i}^{i n}}}{A_{i} h_{i}^{k}}\left(\frac{h_{i}^{k}}{h_{i}^{k f}}\right)^{5 / 3} \quad$ if $h_{i}^{k f}<h_{i}^{k}(28$

A series solution of Eqs. (28a) and (28b) is possible, but a good approximation can also be found with a smaller computational time by setting:

$\xi=\frac{\exp \left(c_{1} \tau\right)+c_{2}}{\exp \left(c_{1} \tau\right)+c_{3}} \quad$ if $h_{i}^{k f}>h_{i}^{k}$

$\xi=1+\left(\xi_{f}-1\right) \frac{\exp \left(c_{1} \tau\right)+c_{2}}{\exp \left(c_{1} \tau\right)+c_{3}} \quad$ if $h_{i}^{k f}<h_{i}^{k}$

with a proper choice of the $c_{1}, c_{2}$ and $c_{3}$ coefficients. Using any $c_{3}$ value it is possible to match the initial value $\xi_{0}$ and its first derivative $\xi_{0}^{\prime}$ by setting:

$c_{2}=\xi_{0}\left(1+c_{3}\right)-1, \quad c_{1}=\xi_{0}^{\prime} \frac{\left(1+c_{3}\right)^{2}}{\left(c_{3}-c_{2}\right)} \quad$ if $h_{i}^{k f}>h_{i}^{k}$

$c_{2}=-1, \quad c_{1}=\xi_{0}^{\prime} \frac{\left(1+c_{3}\right)}{\left(\xi_{f}-1\right)} \quad$ if $h_{i}^{k f}<h_{i}^{k}$

Observe that functions (29a) and (29b) always match the initial and the asymptotic values of the real solution and guarantee its time second order convergence for any given $c_{3}$ value. The $c_{3}$ coefficient affects the maximum error that is obtained according to functions (29a) and (29b) using different time step sizes. This optimum depends on $\xi_{0}$ for case (a) and on $\xi_{f}$ for case (b). The optimum coefficients have been computed numerically for different possible $\xi_{0}$ and $\xi_{f}$ values by comparing functions (29a) and (29b) with a numerical solution computed using a very small time step. See in Table 1 and in Fig. 7 the computed optimum $c_{3}$ values.

See in Figs. 8a and 8b the numerical solution of Eqs. (28a) and (28b) in the case of respectively $\xi_{0}=0$ and $\xi_{f}=0$, compared with the semi-analytical solutions (29a) and (29b) corresponding to the optimal $c_{3}$ values (respectively 0.7469 and -0.8171 ). The maximum error computed with the initial conditions $\xi_{0}=0$, for $h_{i}^{k f}>h_{i}^{k}$, or $\xi_{f}=0$, for $h_{i}^{k f}<h_{i}^{k}$, is the worse one and it is smaller

Table 1

$c_{3}$ coefficient that provides the smallest error for any possible $\tau$ value.

\begin{tabular}{lll}
\hline$\xi, \xi_{f}$ & $\begin{array}{l}h_{i}^{k f}>h_{i}^{k} \\
c_{3}\end{array}$ & $\begin{array}{c}h_{i}^{k f}<h_{i}^{k} \\
c_{3}\end{array}$ \\
\hline 0.0 & 0.746910 & -0.817120 \\
0.1 & 0.579450 & -0.605480 \\
0.2 & 0.460290 & -0.473940 \\
0.3 & 0.366280 & -0.374090 \\
0.4 & 0.288870 & -0.293430 \\
0.5 & 0.223400 & -0.226030 \\
0.6 & 0.166990 & -0.168420 \\
0.7 & 0.117660 & -0.118360 \\
0.8 & 0.074026 & -0.074301 \\
0.9 & 0.035066 & -0.035129 \\
\hline
\end{tabular}

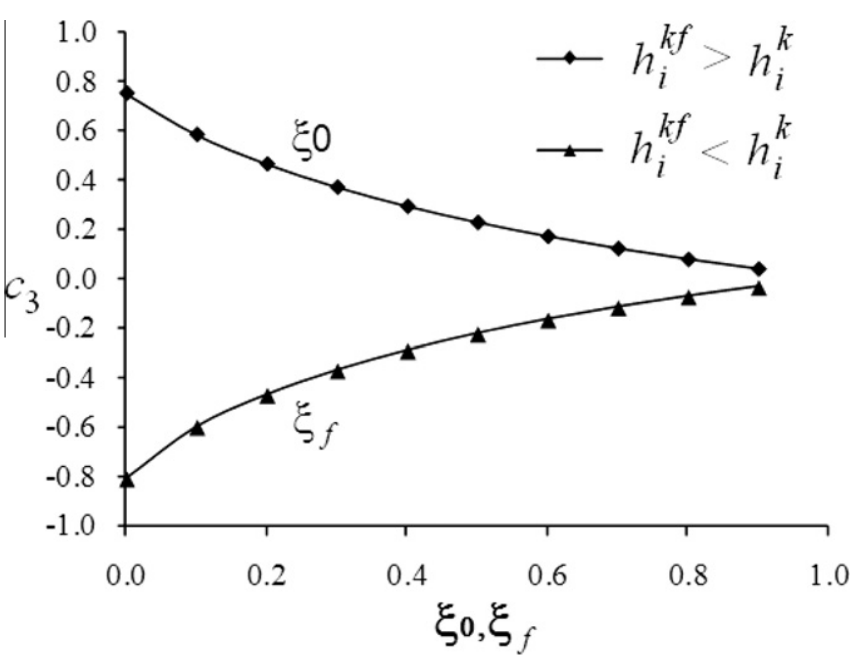

Fig. 7. Function $c_{3}$.

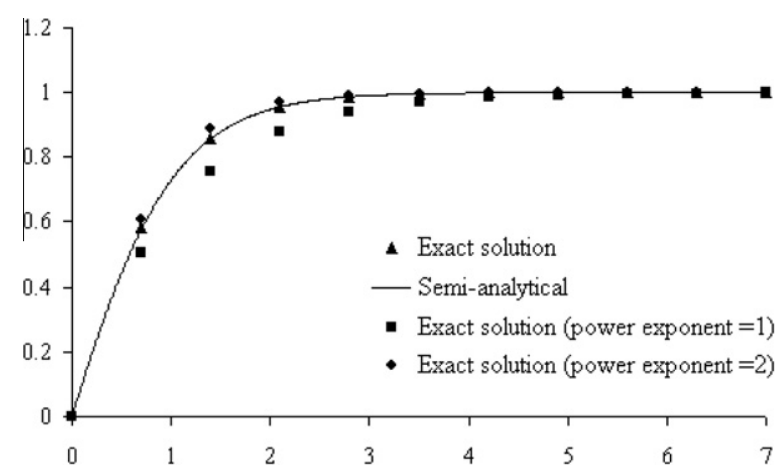

Fig. 8a. Comparison of the solution obtained by the semi-analytical method and the exact solution: case $\xi_{0}=0-h_{i}^{k f}>h_{i}^{k}$.

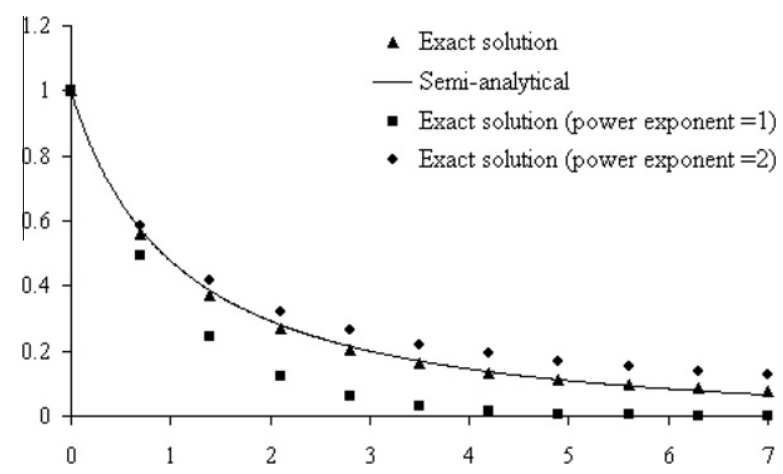

Fig. 8b. Comparison of the solution obtained by the semi-analytical method and the exact solution: case $\xi_{f}=0-h_{i}^{k f}<h_{i}^{k}$.

than $10^{-3}$. See also, in the same figures, the semi-analytical solutions corresponding to $c_{3}=0$ and $c_{3}=1$. These two solutions are equivalent to the analytical closed form solution of Eqs. (28) when the power exponent is approximated respectively to 1 or 2 .

Observe that no special additional treatment for wetting and drying procedure is required in the proposed algorithm. An important feature of Eq. (15), in facts, is that it can be solved also with zero initial water depth value. Unless the spatial water level gradient at time level $k$ is close to zero around the cell, the kinematic wave of the prediction problem propagates beyond the dry cell and this allows the use of Courant number greater than one. 
Observe that the semi-analytical solution also exists in the case $\xi_{0}=0\left(h_{i}^{k f}>h_{i}^{k}\right)$. If, after the solution of the prediction step, $h_{i}^{k m}=0$, the corresponding extra-diagonal terms of the system matrix are zero as well and zero fluxes are computed in all the elements surrounding cell $i$.

\section{Local head losses due to vertical walls}

Diffusive models are fully suitable for the reconstruction of water level profiles and of the vertically averaged velocities in most of the computational domain, for the simulation of natural floods. In some cases and for some special purposes, where vorticity or vertical velocity components have to be estimated, diffusive models are no more adequate, but in other cases it is possible to account for the effect of downstream local energy losses by means of a fictitious change of the Manning coefficient. One of these cases is the restriction of a river cross section given by vertical or subvertical walls.

Modelling a river stream flow with a diffusive 2D model is equivalent to compute the total discharge as the integral of the discharge per unit width, computed along the direction transverse to the flow with a vertically averaged velocity that is different on each element crossed by the vertical section. The same methodology is applied by most of the 1D models using the so called Divided Channel Method (DCM), along with making the further assumption of a single energy slope inside the section [51]. The inconvenient of this approach is that it underestimates the flow resistance in the case of vertical or subvertical walls, because the velocity reduction occurring near the wall is not accounted for by the model.

The reduction of conveyance capacity, caused by walls, can be restored by giving to the elements inside the restriction an equivalent roughness coefficient $n_{p}$, different from the natural one. $n_{p}$ is the roughness coefficient the provides, in steady-state flow condition, the same discharge per unit width $q_{p}$ calculated taking into account the shear stress induced by the walls.

The effective conveyance capacity is estimated at the beginning of the time marching procedure, applying the Interacting Divided Channel Method (IDCM) proposed by Huthoff et al. [25]. The IDCM is based on a parameterization of the interface stress between adjacent flow compartments; assuming steady-state and 1D flow the authors have proposed the following equations to compute the flow velocity per unit bed slope in the compartments (see Fig. 9):

$\rho g A_{j}=\rho f_{j} P_{j} V_{j}^{2}+h_{j-1 / 2} \cdot \tau_{j-1 / 2}+h_{j+1 / 2} \cdot \tau_{j+1 / 2}$

where $\rho$ is the density, $g$ is the gravity acceleration, $A_{j}$ is the compartment area, $h_{j-1 / 2}$ refers to the interface on the left and $h_{j+1 / 2}$ to the interface on the right of compartment $j$. The corresponding shear stress and the dimensionless bed roughness are

$\tau_{j+1 / 2}=\frac{1}{2} \alpha \rho\left(V_{j+1}^{2}-V_{j}^{2}\right)$

$f_{j}=\frac{g n_{j}^{2}}{R_{j}^{1 / 3}}$

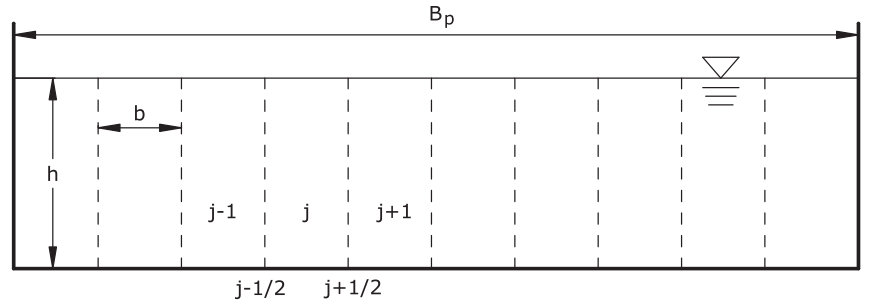

Fig. 9. Subdivision of the cross-section and notations. where $\alpha$ is a dimensionless interface coefficient empirically set equal to 0.02 [25], $n_{j}$ is the natural Manning's roughness coefficient and $R_{j}$ is the compartment hydraulic radius. Observe that, if the interface coefficient is set equal to 0 , namely the lateral momentum transfer is neglected, Eq. (31) is equivalent to the standard DCM for a large enough number of compartments. This is because, if the compartment width is small enough, also the velocity and the discharge per unit width in the two lateral compartments become negligible, but this affects the velocity distribution inside the entire section only if $\alpha \neq 0$. In Fig. 10 we can see how the mean hydraulic radius, defined as:

$R=\left(\frac{q}{\sigma \sqrt{i}}\right)^{3 / 2}$

changes for normal flow inside a rectangular section according to the choice of the $\alpha$ coefficient. The third curve of the same graph shows the hydraulic radius computed as the simple ratio between the total cross section area and its wetted perimeter. We can observe that this value is intermediate between those estimated assuming a compartment segmentation and $\alpha=0$ or $\alpha=0.02$.

Before starting the marching in time simulation, the discharge per unit slope versus piezometric level in the $p$ th restriction is computed as:

$Q_{p}=\sum_{j} b_{j} V_{j} h_{j}$

At the beginning of each time step $k$, the equivalent roughness coefficient $n_{p}$ is be computed as:

$n_{p}=\frac{\left(h^{k}\right)^{5 / 3} B_{p}}{Q_{p}\left(h^{k}\right)}$

where $B_{p}$ is the restriction width.

Inside cross section restrictions, like between the piles of a bridge, a major head loss can also be given by the existence of an hydraulic jump. Once again, it is possible to take into account the jump effects by an artificial increase of the natural Manning coefficient, after the jump existence is checked out at each time level. The jump existence is tested by neglecting the transition effects and comparing the uniform flow energy upstream the restriction

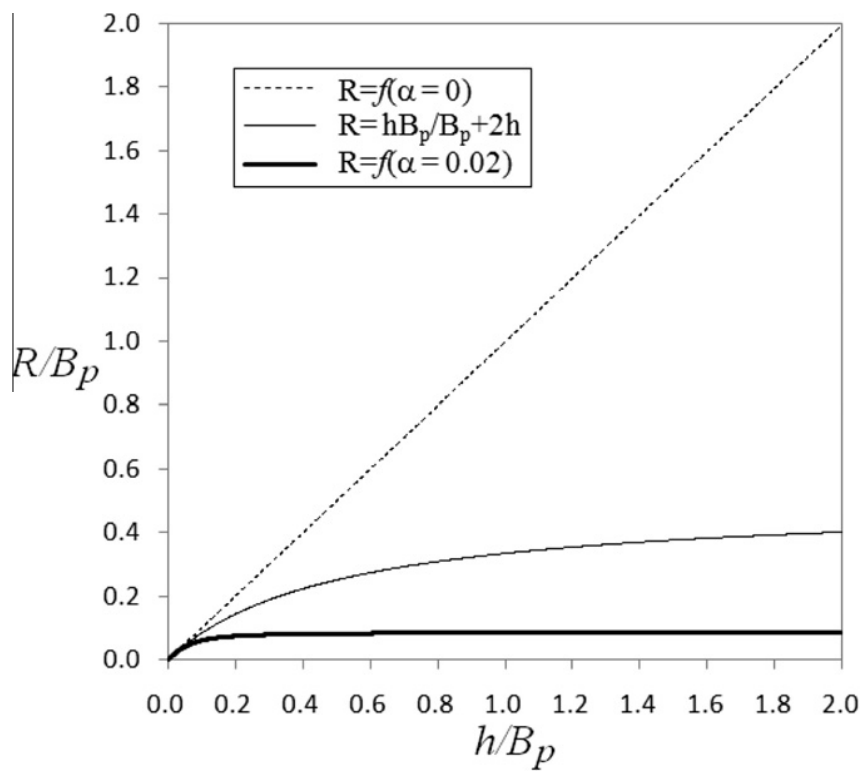

Fig. 10. Dimensionless hydraulic radius. 
with the minimum possible energy inside the restriction, for equal total discharge. The upstream uniform flow energy $E_{0}$ is equal to:

$E_{0}=\left(\frac{n \cdot r_{r} \cdot q_{p}}{\sqrt{i}}\right)^{3 / 5}+\left(\frac{i \cdot\left(r_{r} \cdot q_{p}\right)^{4 / 3}}{n^{2}(2 g)^{5 / 3}}\right)^{3 / 5}$

where $n$ is the natural Manning roughness coefficient, $r_{r}$ is the contraction coefficient given by the ratio between the total width inside and upstream the section restriction and $q_{p}$ is the discharge per unit width computed at beginning of time level $k$ in a given element inside the restriction. The minimum critical energy is equal to:

$E_{k}=\frac{3}{2}\left(\frac{q_{p}^{2}}{g}\right)^{1 / 3}$

Observe in Fig. 11 the comparison between the uniform flow and the critical state energy, as function of parameters $r_{r}$ and $i$.

The uniform flow energy is smaller than the critical state energy, with subsequent hydraulic jump existence, only within a restricted slope range. Similarly, one can see by comparing the $q_{p}$ exponents on the rhs of Eqs. (37) and (38) that uniform flow energy can be greater than critical state energy for very small or very large discharge values.

This implies that temporal discontinuity of the profile may appear upstream the restriction during very slow increment of the discharge $q_{p}$ in quasi-steady flow conditions, even without a wave front spatial propagation.

If the uniform flow energy inside the restriction is too small to allow the computed discharge, a total head loss reduction will occur before the restriction, and the saved energy will be dissipated with an hydraulic jump. The flow depth $h_{m}$ in the section immediately upstream the restriction can then be computed using the following rating curve, for fixed minimum $E_{k}$ energy calculated by Eq. (38):

$h_{m}^{3}-E_{k} h_{m}^{2}=-\frac{q_{m}^{2}}{2 g}$

where $q_{m}$ is the upstream discharge per unit width immediately before the restriction.

The fictitious roughness coefficient $n_{r}$ is computed in order to obtain a total head loss inside the restriction equal to the difference between the minimum upstream energy and the piezometric level $H_{v}$ immediately downstream the restriction. This can be done by integrating the following diffusive momentum equation:

$\frac{d H}{d x}=-\frac{n_{r}^{2} q_{p}^{2}}{(H-z)^{10 / 3}}$

to get:

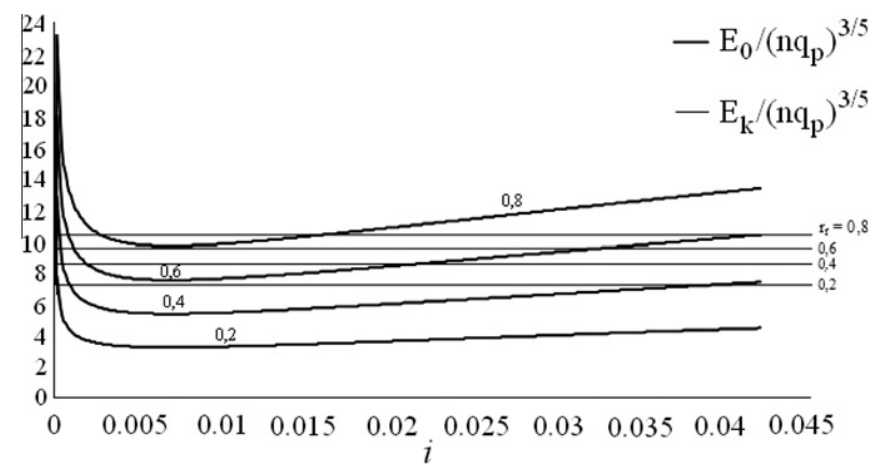

Fig. 11. Dimensionless energy $E_{0} /\left(n q_{p}\right)^{3 / 5}$ and $E_{k} /\left(n q_{p}\right)^{3 / 5}$ versus parameters $i$ and $r_{r}$.

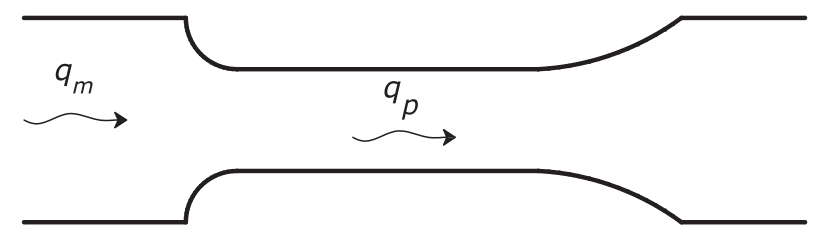

(a) Plan

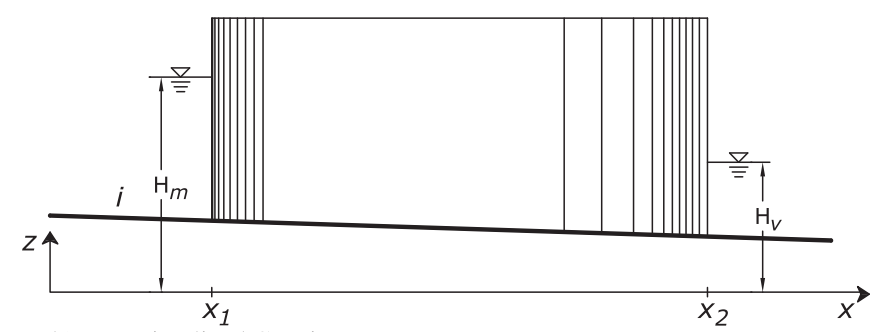

(b) Longitudinal Section

Fig. 12. Restriction scheme and notation.

$h_{m}+z_{m}-H_{v}=n_{r}^{2} \int_{x_{1}}^{x_{2}} \frac{q_{p}^{2}}{(H-z)^{10 / 3}} d x$

where $x_{1}$ and $x_{2}$ (see Fig. 12) are the initial and final restriction abscissa. The fictitious Manning coefficient $n_{r}$ is computed as the root of Eq. (41).

If the restriction width is constant, computation can be simplified by assuming a constant average bed elevation $z_{a}$ inside the integral, to get:

$n_{r}^{2}=\frac{7\left(h_{m}+z_{m}-H_{v}\right)}{3 q_{p}^{2}}\left[\frac{1}{\left(H_{m}-z_{a}\right)^{7 / 3}}-\frac{1}{\left(H_{v}-z_{a}\right)^{7 / 3}}\right]^{-1}$

The procedure has been compared with literature results. Tang et al. [53] compare the discharge hydrographs computed at the end of a rectangular channel using three different types of 1D Muskingum-Cunge models: two VPMC (Variable Parameters Muskingum-Cunge) and one CPMC (Costant Parameters MuskingumCunge) methods. The channel has $50 \mathrm{~m}$ width, $100 \mathrm{~km}$ total length, $0.025 \%$ bed slope and $0.035 \mathrm{~s} / \mathrm{m}^{1 / 3}$ Manning's coefficient.

The computational domain has been discretized using a mesh with right-angle triangles and two parallel lines of 2001 nodes, each one with a distance of $50 \mathrm{~m}$ from the next one. The flow depth corresponding to a constant discharge of $100 \mathrm{~m}^{3} / \mathrm{s}$ has been used as initial condition. The Dirichlet boundary condition was applied to the two downstream final nodes. Even if the depth/width ratio is smaller than 1:10, we can see in Fig. 13 that the routed discharge hydrograph is strongly affected by the choice of the $\alpha$ coefficient in Eq. (32), that is by the type of velocity distribution assumed in the vertical cross sections.

In the case of interface coefficient $\alpha$ equal to 0 (equivalent to using the DCM method), the routed discharge hydrograph computed by the proposed MAST-2D diffusive model is equal to the hydrograph obtained by the DORA 1D diffusive model [38] adopting the DCM method and has a peak value higher than that computed by the Muskingum-Cunge models (Fig. 13). This seems correct, because assuming $\alpha=0$ is equivalent to neglect the wall effects and underestimate the hydraulic resistance. If the lateral momentum transfer is not neglected $(\alpha=0.02)$, the results show a greater peak reduction with respect to the Muskingum-Cunge model, that compute the hydraulic resistance as function of the hydraulic radius of the overall section. This is consistent with the result already shown in Fig. 10, where different ways of computing the average hydraulic radius in normal flow conditions have been compared. 


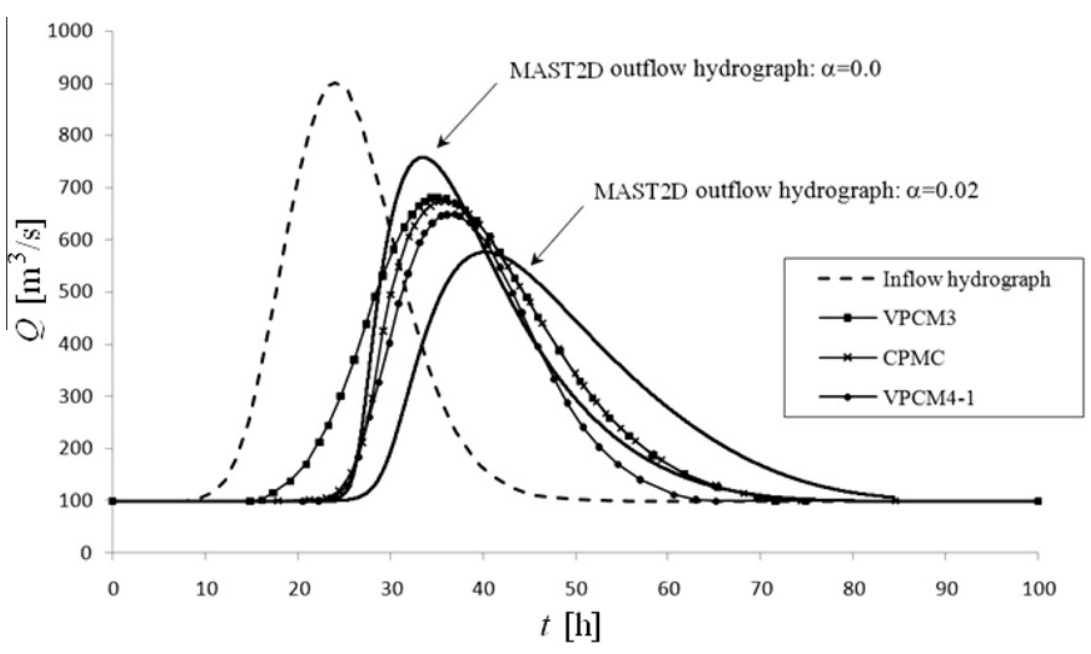

Fig. 13. Outflow discharge hydrographs computed by the proposed and the Muskingum-Cunge models.

\section{Numerical experiments}

In this section, we present five numerical tests for the model validation in the most general 2D-case.

\subsection{Test 1. Stability with regard to the Courant number}

In the first test we investigate the stability of the model results against the size of the element CFL number, computed as:

$\mathrm{CFL}=\frac{V_{e} \cdot \Delta t}{\sqrt{A_{e}}}$ where $A_{e}$ and $V_{e}$ are respectively the element area and the velocity computed in the center of the element assuming a water depth equal to the average value at the element nodes.

A symmetric square $[10,000 \mathrm{~m} \times 10,000 \mathrm{~m}]$ domain has been discretized with the unstructured Delaunay computational mesh shown in Fig. 14, with 1961 nodes and 3758 elements. In order to magnify the irregularity of the mesh, three internal boundaries have been assigned to the domain. The open source mesh generator NETGEN $[37,46]$ has been used to generate the initial mesh. The algorithm presented in Section 5 has been finally used to obtain the final mesh and to guarantee the Generalized Delaunay property, without changing the assigned internal and external

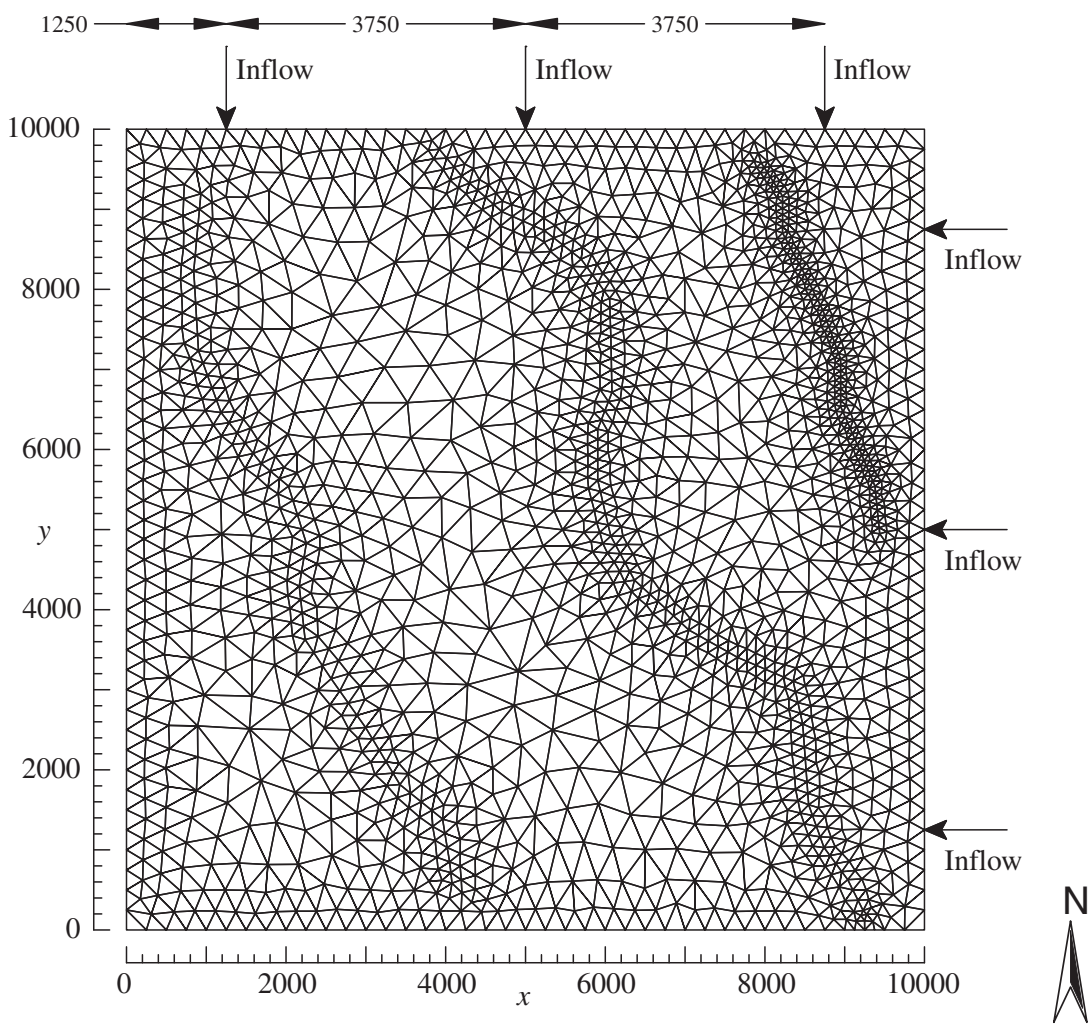

Fig. 14. Test 1. Computational mesh. 
boundaries. The Manning coefficient is $0.025 \mathrm{~s} / \mathrm{m}^{1 / 3}$, the bottom elevation of point $(0,0)$ is equal to zero and the bottom slope is 0.001 in both $x$ and $y$ directions. A constant zero water depth has been assigned on the East and South external domain sides as Dirichlet boundary conditions. A symmetric triangular inflow hydrograph in the boundary inflow nodes shown in Fig. 14, with peak discharge equal to $2500 \mathrm{~m}^{3} / \mathrm{s}$ and peak time equal to $8 \mathrm{~h}$, has been given as Neumann upstream boundary condition at the North and West side. Initial condition is $h_{0}=0$.

Five simulations with different time step size have been carried out on the same mesh. Table 2 shows the time step size $\Delta t$, the maximum CFL value calculated according to Eq. (43) in the domain during the entire simulation, the mean CPU time per computational node and per iteration and the relative error $\varepsilon$ of the solution at the peak time, between the 1 st and $m$ th simulation, calculated as:

$\varepsilon_{m}=\sqrt{\frac{\left(h^{m}-h^{r e f}\right)^{2}}{\left(h^{r e f}\right)^{2}}}$

where $h^{m}$ is the maximum water depth computed in node $i$ during the $m$ th simulation and $h^{\text {ref }}$ is the same water depth computed during the $1 \mathrm{st}$ simulation with the minimum time step size. Node $i$ is located in the center of the domain.

Observe that the stability and accuracy of the model is obtained also for very large values of the CFL number. In this test case, if CFL is less than 21.5 , the maximum relative error $\varepsilon$ is less than $1 \%$. Moreover, the increase in the CFL number does not lead to any increase in the mean CPU time. If the CFL number is greater than 70 the stability of the model is guaranteed, but in the transient solution there is a numerical diffusion, leading to a flattening and a deformation of the water depth curve (Fig. 15).

Table 2

Test 1. max. CFL numbers, errors and mean CPU times.

\begin{tabular}{lrcll}
\hline Simulation & $\Delta t[\mathrm{~s}]$ & Max CFL & Mean CPU times $[\mathrm{s}]$ & $\varepsilon$ \\
\hline 1 & 15 & 0.54 & $1.06 \mathrm{E}-02$ & - \\
2 & 40 & 1.44 & $1.05 \mathrm{E}-02$ & $1.15 \mathrm{E}-04$ \\
3 & 150 & 5.38 & $1.11 \mathrm{E}-02$ & $1.29 \mathrm{E}-03$ \\
4 & 600 & 21.5 & $1.15 \mathrm{E}-02$ & $8.73 \mathrm{E}-03$ \\
5 & 2000 & 73.54 & $1.31 \mathrm{E}-02$ & $3.48 \mathrm{E}-02$ \\
\hline
\end{tabular}

To test the robustness of the model with respect to the mesh irregularity, the solution of the second simulation $(\Delta t=40 \mathrm{~s})$ at the peak time $t=32,000 \mathrm{~s}$ (see Fig. 15) has been compared with the results obtained over an unstructured mildly distorted mesh with 1931 nodes and 3700 elements (see Fig. 16a). Fig. 16b shows the absolute values of the relative difference between water levels calculated over both the mildly and highly unstructured meshes. The relative difference has been computed as:

$\Delta h(x, y)=\frac{h^{r e}-h^{i r}}{\hat{h}^{r e}}$

where $h^{r e}$ and $h^{i r}$ are the water depths computed with respectively the more and less regular mesh and $\hat{h}^{r e}$ is the average water depth value computed with the regular mesh over all the domain. Observe that the relative difference is less than $1 \%$ over most of the computational domain and the larger differences are close to the

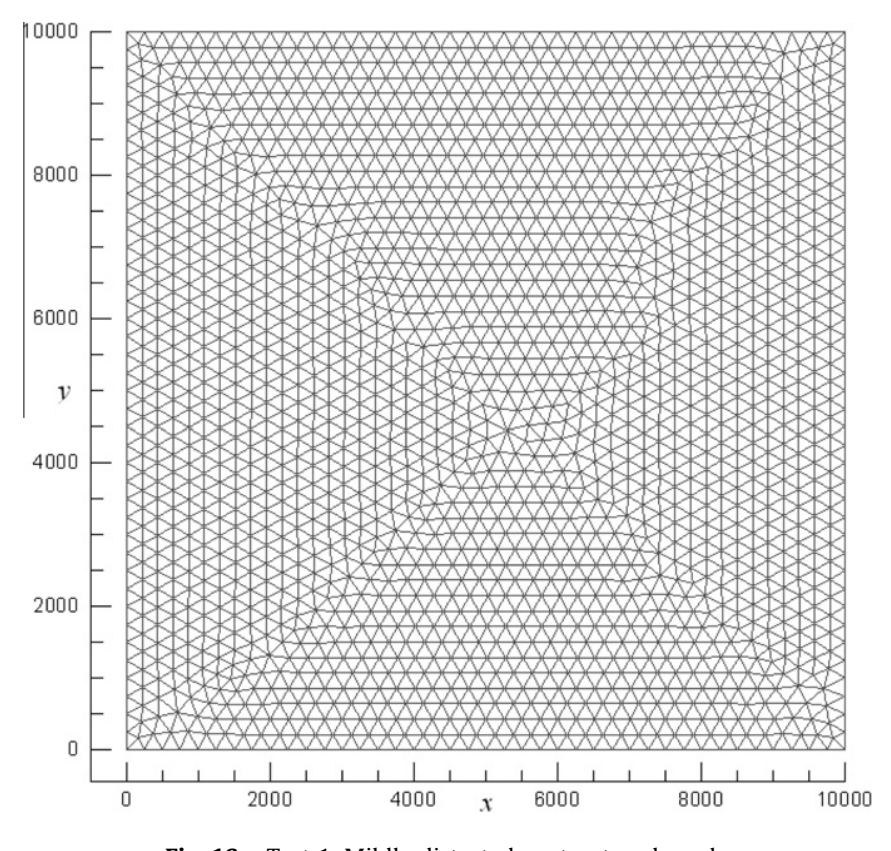

Fig. 16a. Test 1. Mildly distorted unstructured mesh.

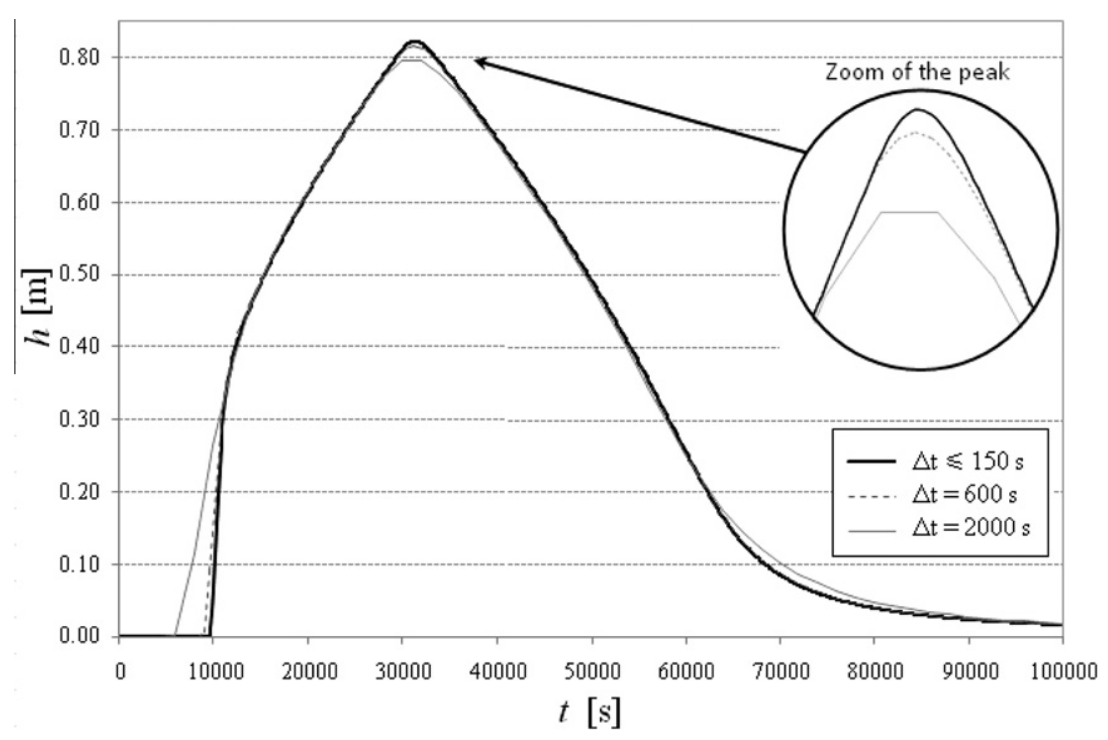

Fig. 15. Test 1. Water depth curve in the center of the domain. 


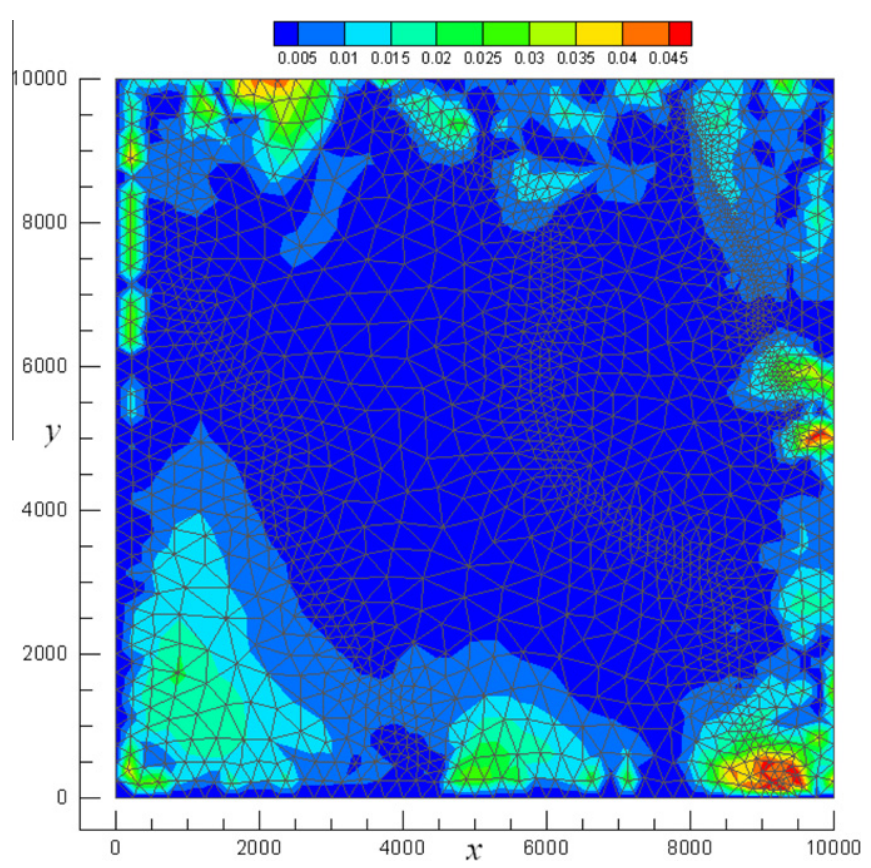

Fig. 16b. Test 1 . Absolute value of the relative difference between water surface calculated on the mildly and highly distorted mesh.

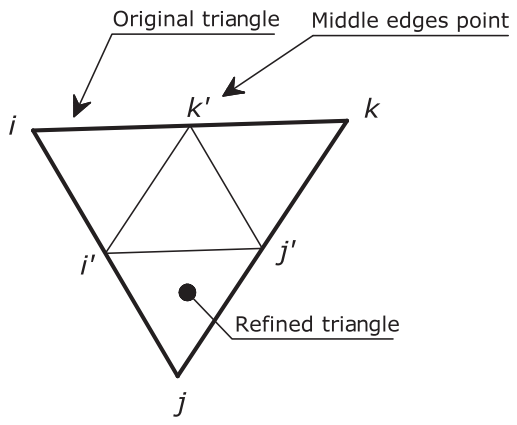

Fig. 17. Test 2. Refinement scheme.

boundaries, where the solution is strongly affected by the element size, but the same difference is very small around the internal boundaries, where the mesh is more distorted.

\subsection{Test 2. Computational performance investigation}

In the second numerical experiment, the computational performance of the proposed model has been investigated, using the same mesh of the previous experiment (see Fig. 14). The original mesh has been refined by dividing each element in four equal triangles and connecting the midpoints of the three sides of each triangle. See in Fig. 17 the refinement scheme. Three refinement levels have been carried out. After each refinement, the Generalized Delaunay condition was guaranteed by changing the nodes connection according to the same procedure described in Section 5. Moreover, the same boundary and initial conditions as in test 1 have been applied. For the first simulation (coarsest mesh), a time step of $150 \mathrm{~s}$ has been used, with a maximum CFL number equal to 5.38. In order to obtain a CFL number similar in each refined mesh, the time step has been halved at each refinement.

The CPU time of the total simulation, of the convective step and of the diffusive step, has been recorded for each mesh. Table 3 shows the specific CPU times of a single processor Intel Q9400 $2.66 \mathrm{GHz}$, per node and per time step.

The trend of the CPU time versus the number of nodes is different for the prediction and correction steps. The mean specific CPU time for the convective step remains almost constant, with a growth due only to the cells sorting. The specific CPU time for the solution of the linear system associated to the diffusive problem grows very slowly, and the total CPU time is only a bit more than proportional to the number of nodes. The growth rate $\beta$, measured as the exponent of the relationship:

$C P=N^{\beta}$

where $N$ is the number of nodes and $C P$ is the average $\mathrm{CPU}$ time per each time step, is only 1.10 in the proposed test (see Fig. 18).

\subsection{Test 3. The convergence rate}

The third numerical test is focused on the order of convergence of the proposed model. Because test cases to be used for comparison between analytical and numerical solutions in 2D transient conditions are not available, a different procedure has been applied. The following arbitrary analytical solution $H=H(x, y, t)$ is given for Eq. (4), where the source term on its rhs is computed by time and space differentiation of the known $H$ on the lhs of the same Eq. (4),

$H=-0.001 \frac{\sqrt{x^{2}+y^{2}}}{\sqrt{2}}+\frac{h^{*}}{2}\left[\tanh \left(k\left(u t-\sqrt{x^{2}+y^{2}}\right)\right)+1\right]$

where $x$ and $y$ are the domain point coordinates, $t$ is the time and $k$, $u, h^{*}$ are constants equal to $0.0015,1$ and 3 respectively. The analytical solution has been assigned on the same $[10,000 \mathrm{~m} \times 10,000 \mathrm{~m}]$

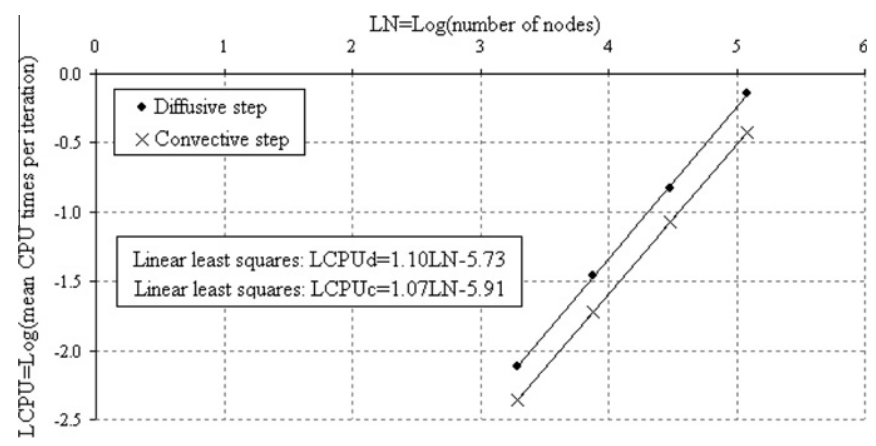

Fig. 18. Test 2. Trend of the CPU times in Eq. (46) required for the solution of the convective and diffusive steps.

Table 3

Test 2. Mean CPU times.

\begin{tabular}{|c|c|c|c|c|}
\hline Refinement & Number of elements & Number of nodes & Mean CPU times (convective) [s] & Mean CPU times (diffusive) [s] \\
\hline 0 & 3758 & 1961 & $2.2310 \mathrm{E}-06$ & $3.9138 \mathrm{E}-06$ \\
\hline 1 & 15,028 & 7677 & $2.4635 \mathrm{E}-06$ & $4.5460 \mathrm{E}-06$ \\
\hline 2 & 60,104 & 30377 & $2.8008 \mathrm{E}-06$ & $4.8425 \mathrm{E}-06$ \\
\hline 3 & 240,400 & 120849 & $3.0772 \mathrm{E}-06$ & $6.0018 \mathrm{E}-06$ \\
\hline
\end{tabular}




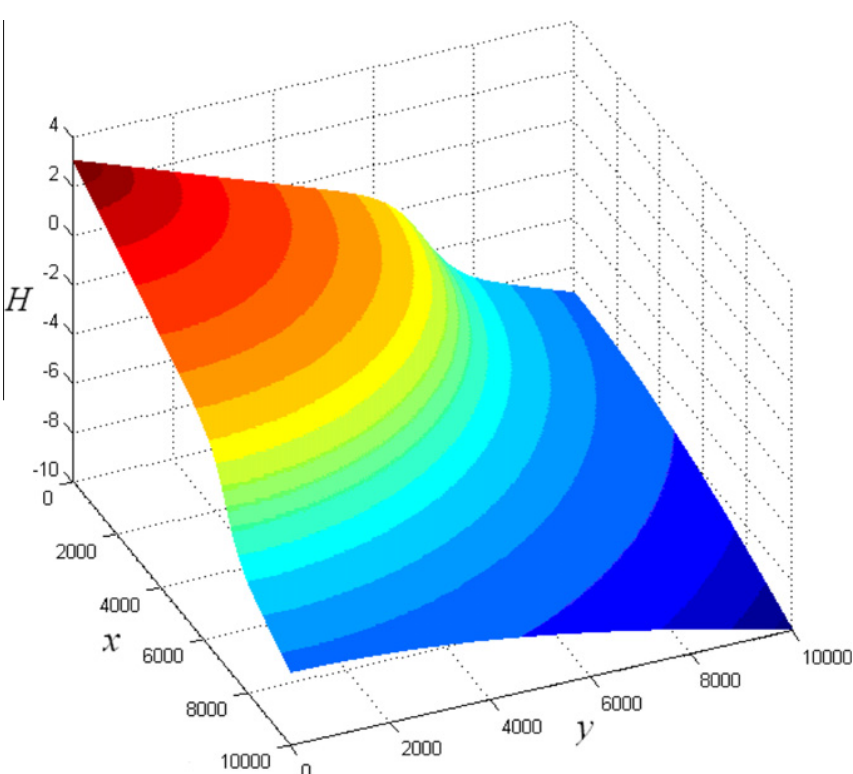

Fig. 19. Test 3. Plot of the exact solution for the piezometric head of Eq. (47) at $t=7000 \mathrm{~s}$.

domain used in the previous tests. The Manning coefficient is $0.025 \mathrm{~s} / \mathrm{m}^{1 / 3}$ and the following bottom elevation $z$ is given:

$z=-0.001 \frac{x+y}{\sqrt{2}}$

Function $H$ in Eq. (47) is shown in Fig. 19 at time $t=7000 \mathrm{~s}$. Given the solution $H$, the source term $p$ in Eq. (4) is computed in each point and at any time by solving the lhs of the same equation. Observe that the proposed function $H$ has zero flux along the South and West sides of the domain and it is assigned as Dirichlet boundary condition in the two other sides.

The same mesh refinement as in test 2 has been used. The time step $\Delta t$ is $100 \mathrm{~s}$ for the coarsest mesh, corresponding to a maximum spatially averaged CFL number equal to 1.74 . The time step
Table 4

Test 3. Convergence rates.

\begin{tabular}{llll}
\hline Refinement & $r_{c}$ upstream node & $r_{c}$ front wave node & $r_{c}$ downstream node \\
\hline 1 & 0.97 & 1.14 & 0.86 \\
2 & 1.13 & 1.83 & 1.00 \\
3 & 1.38 & 1.94 & 1.31 \\
\hline
\end{tabular}

has been halved at each refinement in order to maintain almost constant the CFL number from one refinement to the other.

The relative error on each node and for each mesh has been estimated by Eq. (49a) at the simulation time $t=7000 \mathrm{~s}$ :

$\varepsilon_{m}=\sqrt{\frac{\left(h_{i}^{n}-h_{i}^{a}\right)^{2}}{\left(h_{i}^{a}\right)^{2}}}$

where $h_{i}^{n}$ and $h_{i}^{a}$ are respectively the numerical and the analytical water depth computed in node $i$.

The rate of convergence is defined by comparing the relative errors of two consecutive mesh levels. Assuming the relative error obtained for mesh level $m$ proportional to a power of the linear size of the area of the mean triangle in the mesh, that is:

$\varepsilon_{m}=\left(\sqrt{A_{m}}\right)^{r_{c}}$

where $A_{m}$ is the area of the mean triangles in the mesh refinement level $m$ and $\sqrt{A_{m}}$ represents a measure of its linear size, the rate of convergence $r_{c}$ is computed by comparing the relative errors of two successive refinement levels $m$ and $m+1$ :

$r_{c}=\frac{\log \left(\frac{\varepsilon_{m}}{\varepsilon_{m+1}}\right)}{\log 2}$

Table 4 shows the rates of the convergence calculated at three nodes located respectively on the front, upstream and downstream the advancing wave (see Fig. 20). The results show that: (a) the rate of convergence increases along with the mesh density and this guarantees robustness in the case of very coarse meshes, (b) the asymptotic rate of convergence is higher in the areas with larger piezometric gradients, even greater than 2 , but smaller around

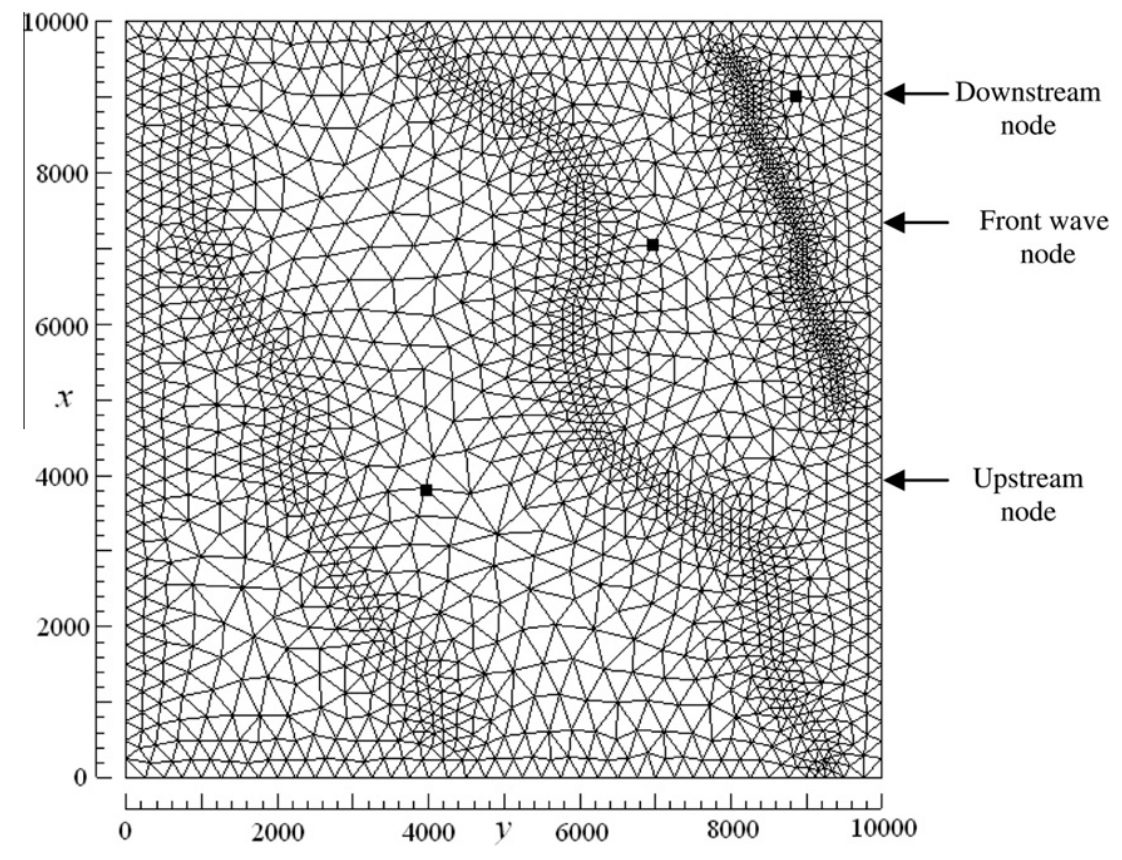

Fig. 20. Test 3. Selected nodes for the convergence rate calculation. 
flatter areas. This can be explained by the proportionality of the fluxes to the root of the norm of the piezometric gradient, that leads to an infinite sensitivity of the fluxes with respect to the piezometric gradient around its zero value.

\subsection{Test 4. Comparison with wetting-drying literature tests}

Gourgue et al. [21] have validated the WD option of their fully dynamic model using a test already proposed by Balzano [9] in one-dimensional form. They proposed a flux limiting WD approach for a DG FE method for the solution of the fully dynamic SW equations. A basin with uniform bottom slope, length $13800 \mathrm{~m}$ in $x$ direction and width $7200 \mathrm{~m}$ in $y$ direction is discretized by an unstructured triangular mesh. The adopted mesh (Fig. 21) has a node density similar to the mesh in [21], with 690 elements and 383 nodes. Manning coefficient is $0.02 \mathrm{~s} / \mathrm{m}^{1 / 3}$, bottom elevation is constant in $y$ direction while the slope $i$ in $x$ direction is 0.00036 .

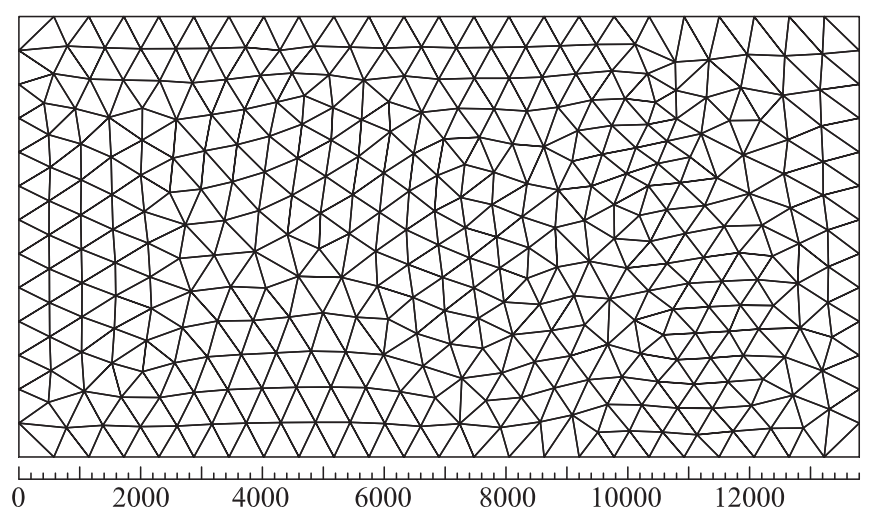

Fig. 21. Test 4. First Balzano test. Computational mesh.

(a)

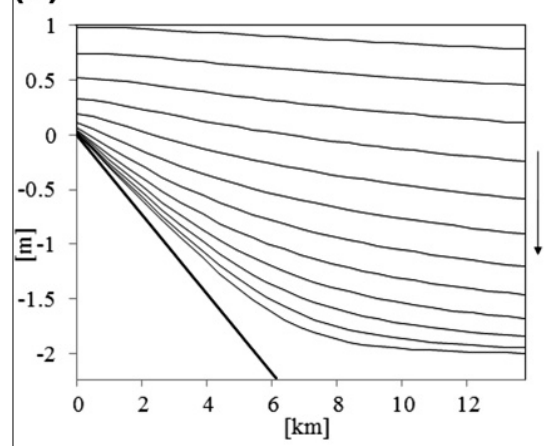

(b)

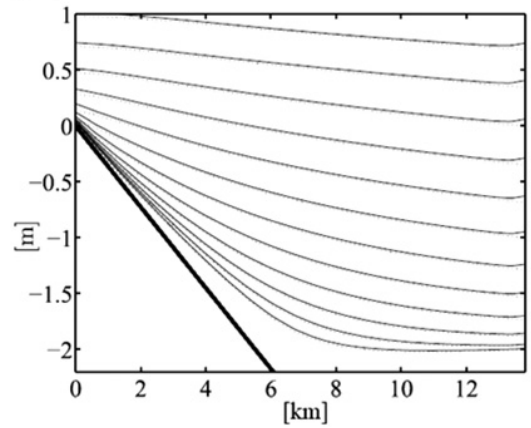

The domain has upstream zero flux boundary, downstream open boundary and the initial condition is piezometric head equal to zero. At the open downstream boundary, a sinusoidal water level variation is assigned. The amplitude and the period of the oscillation are respectively $2 \mathrm{~m}$ and $12 \mathrm{~h}$.

In this case the results of the diffusive model is similar to the results of the complete model, because the time period of the downstream boundary condition is large enough with respect to the travel time of the generated wave, needed to cover the domain length. Observe in Fig. 22 the free surface shape computed by both models every $20 \mathrm{~min}$. The wet-dry limit is very similar at all the investigated times and negative depths, as well as artificial spatial oscillations are missing in both models. The peculiarity of our model is that no special treatment is needed for the elements located near the wet-dry limit.

The proposed numerical model has been tested also for the third Balzano test. The basin contains a small reservoir and the elevation of the bottom is calculated by the following analytical expressions:

$z=-x / 2760$ if $x \leqslant 3600 \mathrm{~m}$ or $x \geqslant 6000 \mathrm{~m}$

$z=x / 2760-60 / 23$ if $3600 \mathrm{~m}<x \leqslant 4800 \mathrm{~m}$

$z=-x / 920+100 / 23$ if $4800 \mathrm{~m}<x<6000 \mathrm{~m}$

The initial condition is a piezometric head equal to $2 \mathrm{~m}$ and the boundary condition is a sinusoidal decay applied at the open boundary. At the open boundary the water depth decreases from $7 \mathrm{~m}$ to $3 \mathrm{~m}$ within $6 \mathrm{~h}$ (half the sinusoidal period). After this period, the water depth at the open boundary is left indefinitely at $3 \mathrm{~m}$ in order to test whether water is leaking through the dry area. The computational mesh is composed of 518 elements and 296 nodes (see Fig. 23). The surface in the reservoir should asymptotically reach an horizontal plane at the level of the local peak of the bathymetry in the pond. Many numerical models fail in representing the water surface profile because water fluxes do not vanish as (c)

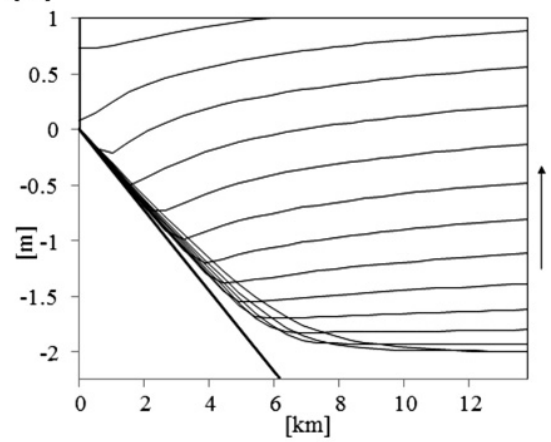

(d)

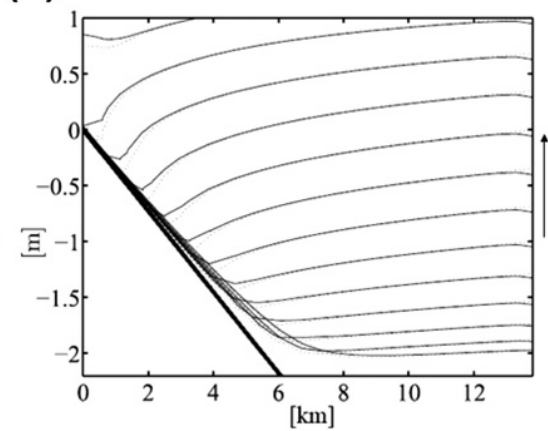

Fig. 22. Test 4. First Balzano test. Water surface every 20 min (thin lines) and bottom channel (thick line). Results of the MAST model (a) and (c); Gourgue et al. [21] model results (b) and (d). 


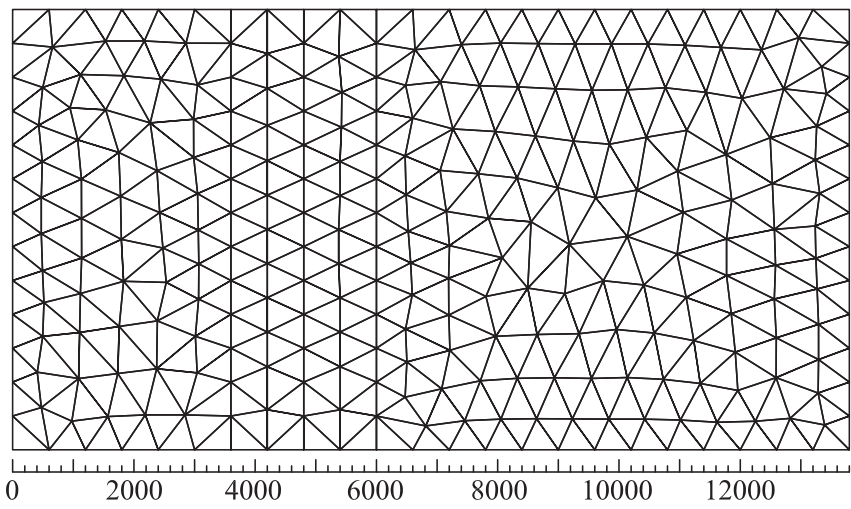

Fig. 23. Test 4. Third Balzano test. Computational mesh.

long as the pressure gradient term operates, even when the mean surface level inside the reservoir is below the local peak of the bathymetry and the pond dries up. When water depth is zero at $x=4800 \mathrm{~m}$ (the right end side of the pond), numerical fluxes computed in the proposed model according to Eq. (22a) vanish. In Fig. 24, the computed water surface profiles are shown and compared with the ones computed by [21] after $100 \mathrm{~h}$. Observe that the expected final water level is perfectly simulated in the reservoir. The reference results have been computed using a similar unstructured mesh (see [21]).

\subsection{Test 5. Comparison with a complete model using an overflow test} case

In this last numerical test the results of the diffusive model have been compared with the results of a fully dynamic model. In this test case both models could be reasonably applied for the water level and velocity computation.

The complete model applied here for comparison is the Godunov-type finite-volume model of BreZo [12,13], that solves the depth-averaged Shallow Water Equations on an unstructured grid of triangular cells. The algorithm of BreZo uses Roe's approximate Riemann solver to compute fluxes, a multidimensional limiter for second-order spatial accuracy and predictor-corrector time stepping for second-order temporal accuracy. The model features a special technique for the treatment of the partially wetted cells based on equations that define exactly the relationship between free surface elevation and water content of each cell (Volume/ Free-surface Relationships, VFRs). These equations are applied at each time step to consistently track fluid volume and the free surface elevation (which is important for flux evaluation) in partially submerged cells. As for the solution algorithm, in the predictor step the solution is updated by solving the SW equations in terms of the primitive variables, to improve the computational efficiency, while in the corrector step the conservative, integral form of the SW equations is solved. Whereas the continuity equation is updated in all cells, the momentum equations are not solved in partially wetted cells (i.e., where at least one of the three nodes is not submerged), where the velocity is set to zero in order to avoid spurious accelerations.

The test case is the steady-state reconstruction of a river flooding. The spatial domain is given by a trapezoidal channel crossing a flat area with a channel depth reduction in the central part of the domain (see in Fig. 25 the plan view and the cross section).

A first unstructured mesh adopted for spatial discretization has 10759 nodes and 20464 elements. In this mesh, only few nodes were used to represent each channel section morphology and zero nodes were located inside the area of the river banks. The
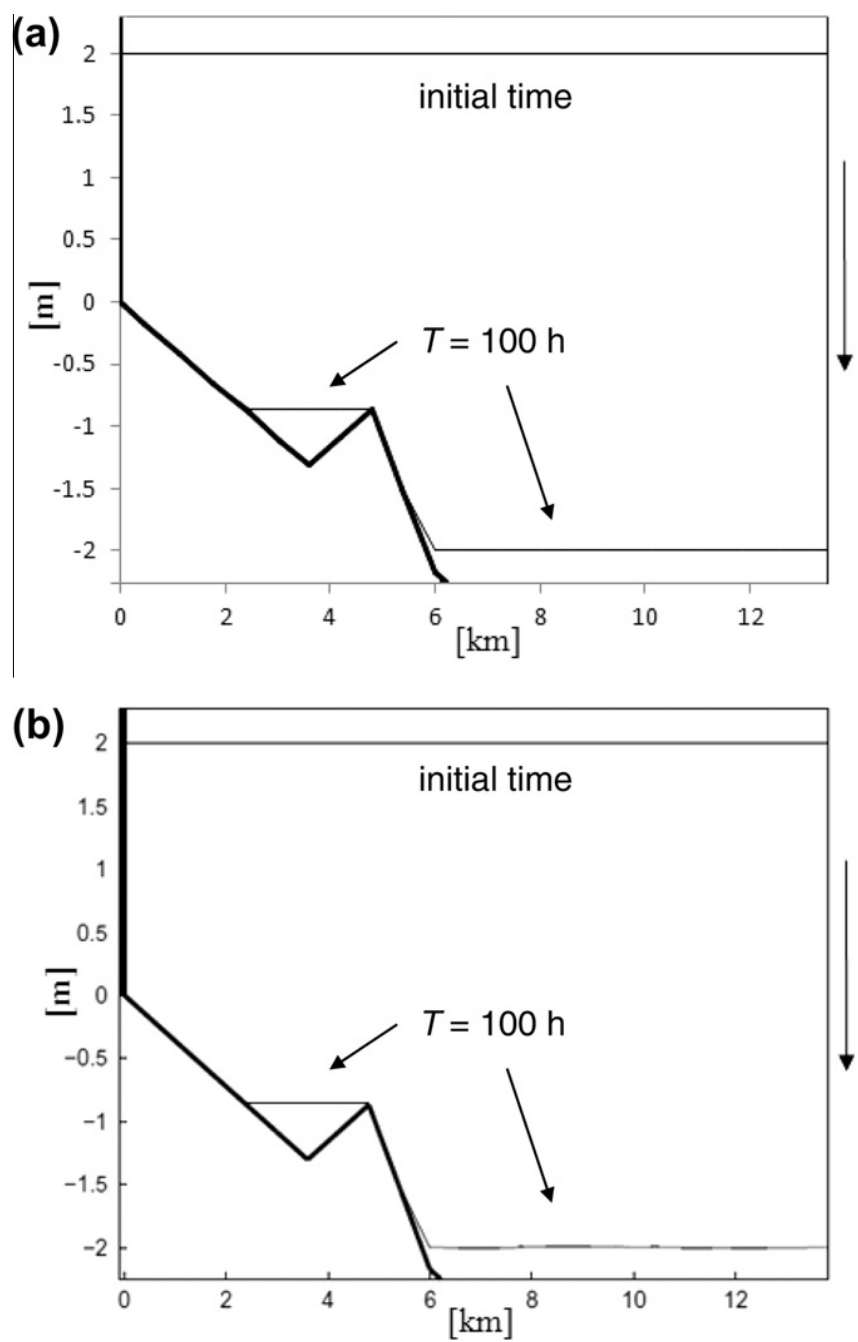

Fig. 24. Test 4. Third Balzano test. Bottom channel (thick line) and position of the water surface at initial time and at equilibrium (thin lines). Results of the MAST model (a); Gourgue et al. [21] model results (b).

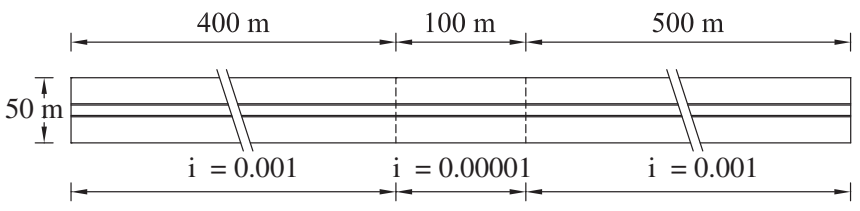

(a) Plan

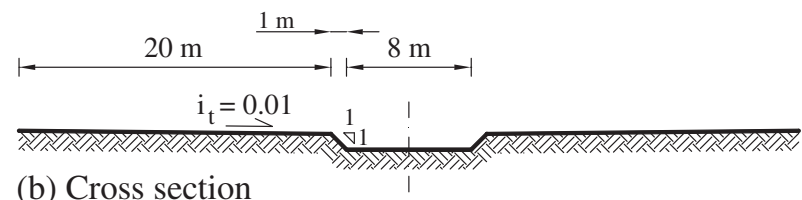

Fig. 25. Test 5. Compound channel.

steady-state discharge is $9 \mathrm{~m}^{3} / \mathrm{s}$, distributed as Neumann boundary condition along the central part of the upstream boundary side (from $y=21 \mathrm{~m}$ to $y=29 \mathrm{~m}$ ); the Manning coefficient is $0.03 \mathrm{~s} / \mathrm{m}^{1 /}$ 3 and the Dirichlet water depth, at the downstream boundary, is equal to normal flow depth corresponding to the upstream condition. Simulation time is $10,000 \mathrm{~s}$, large enough to get steady-state flow in the channel, starting from completely dry bed condition. The adopted $\Delta t$ for the MAST simulations is $5 \mathrm{~s}$ (2000 total time 
iterations), while BreZo scheme adapts the time step size according to the condition $\mathrm{CFL}=0.9 ; \Delta t$ at steady-state conditions is $2.48 \mathrm{~s}$. In the lower part of the channel, flow remains inside the trapezoidal section, while flooding occurs in the central and in the upper parts. The comparison of the solutions obtained with the proposed model and the complete BreZo model shows significant differences in the flooded areas (see Fig. 26).

The computational mesh has been refined as previously explained. Time step for MAST simulations has been reduced to $2.5 \mathrm{~s}$, while the $\Delta t$ at steady-state conditions for BreZo simulations is in this case $0.012 \mathrm{~s}$. After the refinement, the proposed model provides a solution very similar to that obtained with the first mesh (see Fig. 27a). On the other hand, the complete model gives in this case a solution different from the solution obtained with the first mesh and similar to the one computed by the MAST model using the same refined mesh. According to this result, BreZo solution underestimates the flooded area using the coarse mesh (see Fig. 27b).

A first motivation of this difference is that in BreZo the momentum equations are not solved in partially wetted cells, which adds a "numerical" friction in these cells. This effect is noticeable when coarse mesh are used, and quickly decreases as the computational grid is refined, as described in [13]. A second motivation can be found in the different topography represented by the two meshes. Different node elevations correspond to different source term and, for given specific energy, to different water levels. The results of this tests are consistent with the observation of Section 2 and suggest that the stronger stability of the diffusive model can lead to better results in the computation of the water levels even when the approximation of the ground elevation is due not to measurement error, but to the same spatial discretization.

Fig. 28 shows the velocity field computed by the MAST scheme and Fig. 29 shows the scatters from the one computed by the BreZo model. Main differences occur in the flooded areas, eventhough, due to the different scales adopted for the graphics, these differences are very low.

A comparison of the CPU times required by the MAST and BreZo models for the solution of the present test using the finer mesh and a single processor Intel Q9400 $2.66 \mathrm{GHz}$ has been done. Total computational time required by MAST scheme is $2838.52 \mathrm{~s}(13 \%$ for the solution of the prediction step and $87 \%$ for the solution of the correction step). Computational time of the BreZo model is approximately 39 times higher.

The water depths in two nodes of the bank (see Fig. 30) computed using the coarse mesh along the time are shown in Fig. 31. Observe that numerical oscillations never occur and the diffusive correction $\eta$ obtained by the solution of Eq. (11) quickly converges to zero.

\subsection{Test 6. Application to a real case: Venturi channel of Imera river} (Sicily)

The proposed model has been applied for the simulation of the backwater profile caused by the Venturi channel located in the southern part of the Imera river, in Sicily. Fig. 32a shows the geometry of the structure. Fig. 32b shows the vertical walls of the restriction immediately after a flood event and the maximum level

(a)

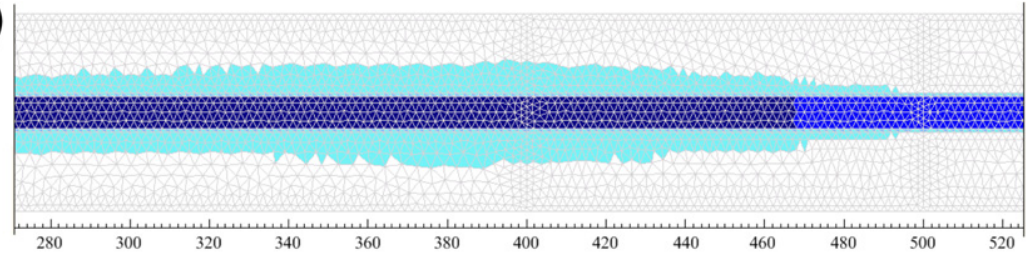

(b)

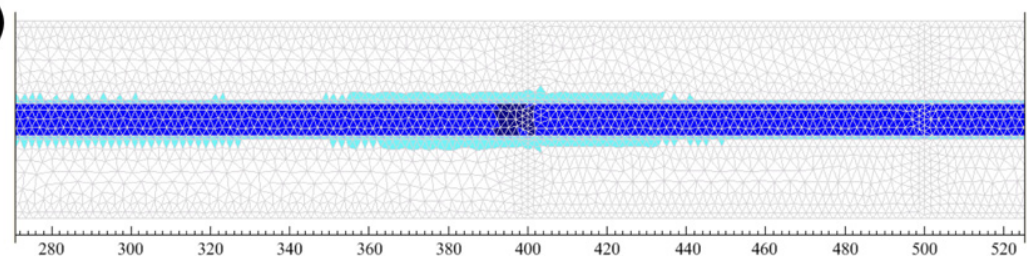

Fig. 26. Test 5. Steady-state water depth upstream the flat area. (a) MAST model and (b) BreZo model [12] (coarse mesh).

(a)

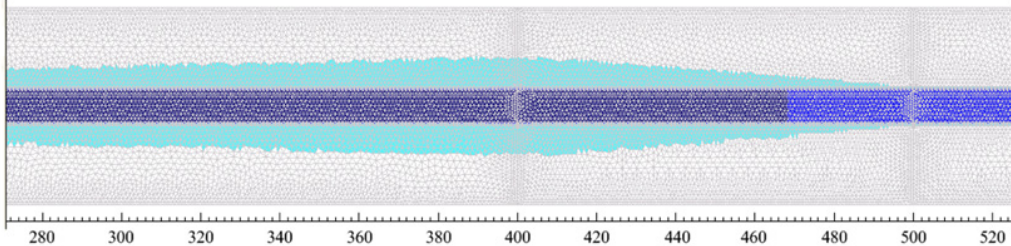

(b)
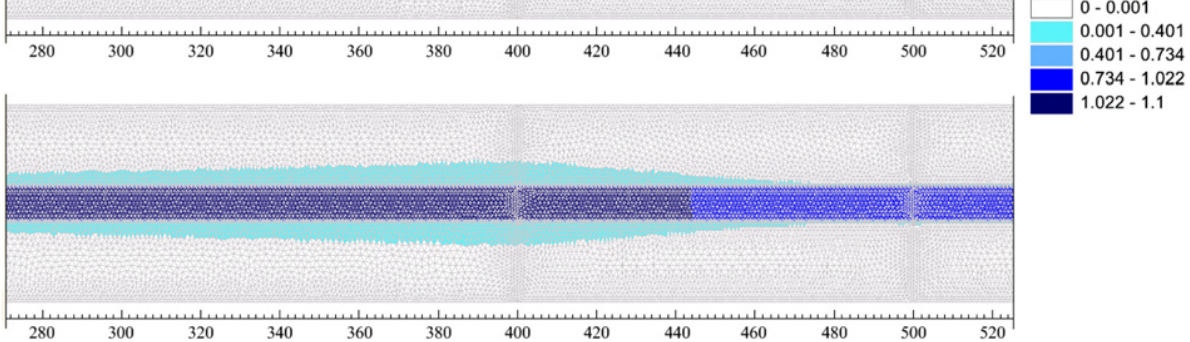
$0.401-0.734$ $0.734-1.022$ $1.022-1.1$

Fig. 27. Test 5. Steady-state water depth upstream the flat area. (a) MAST model and (b) BreZo model [12] (refined mesh). 


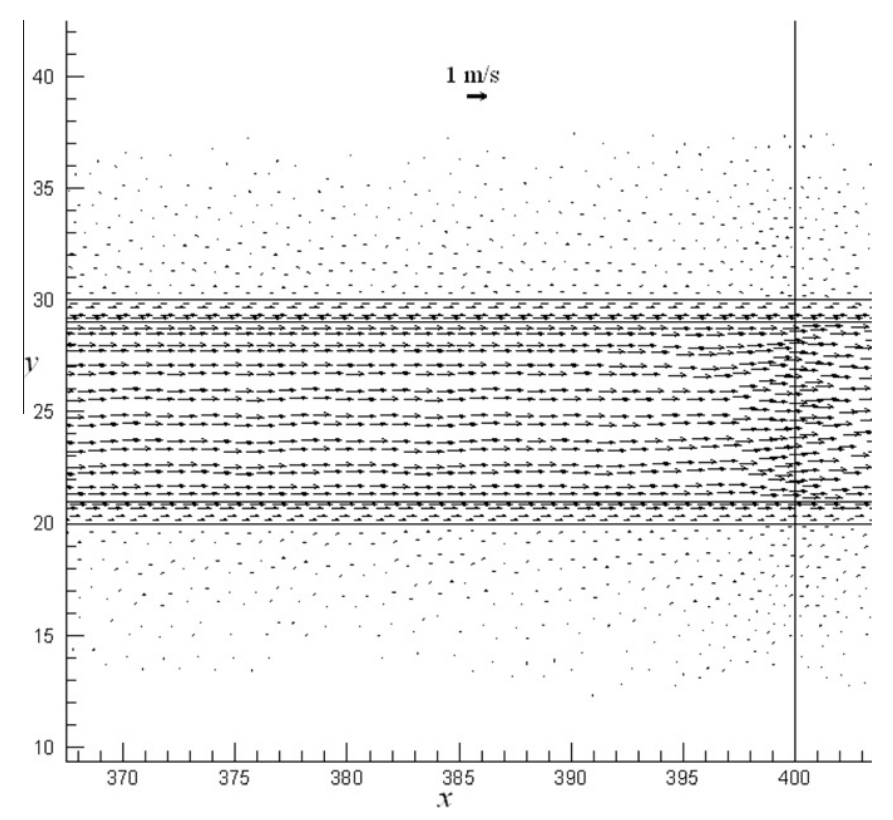

Fig. 28. Test 5 . Detail of velocity field in the refined mesh calculated by MAST model.

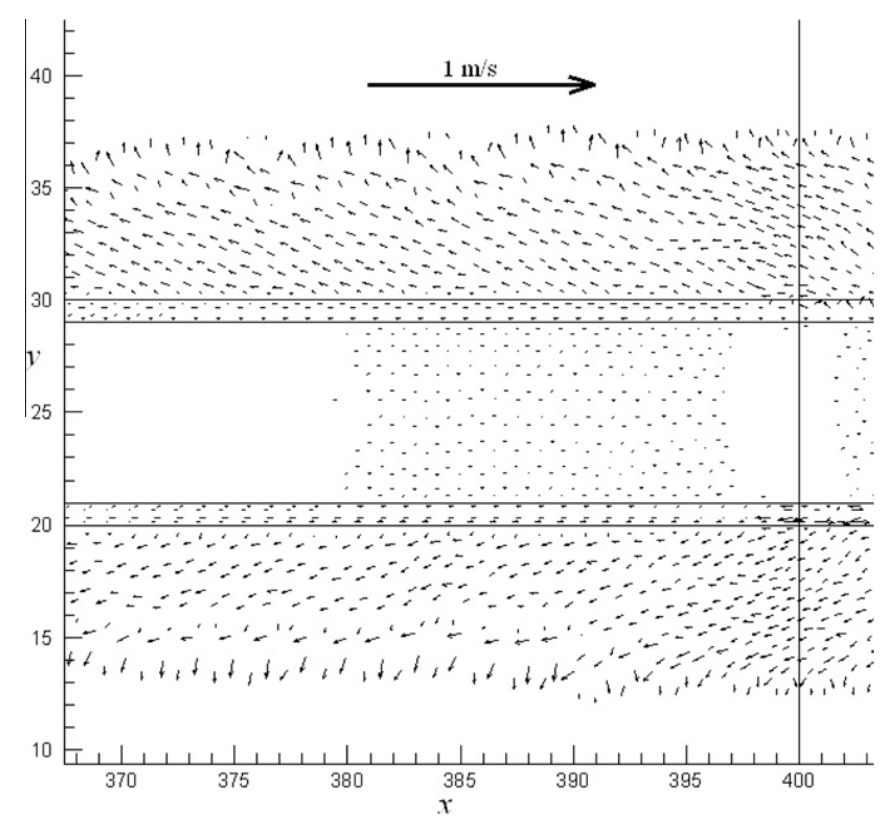

Fig. 29. Test 5 . Detail of the scatters between velocity fields calculated by MAST model and by BreZo model (refined mesh).

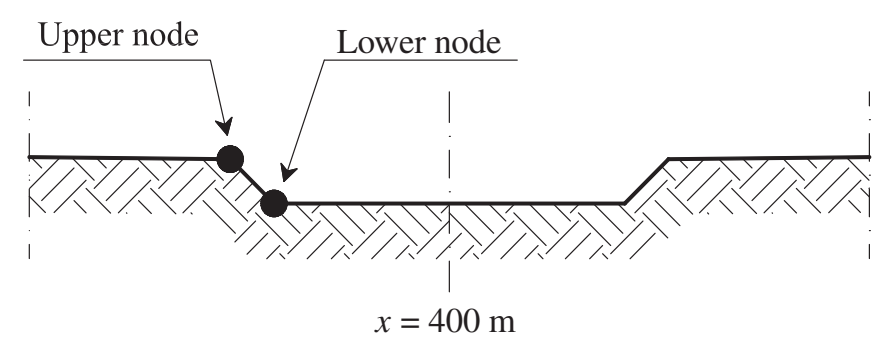

Fig. 30. Test 5. Monitored nodes position.

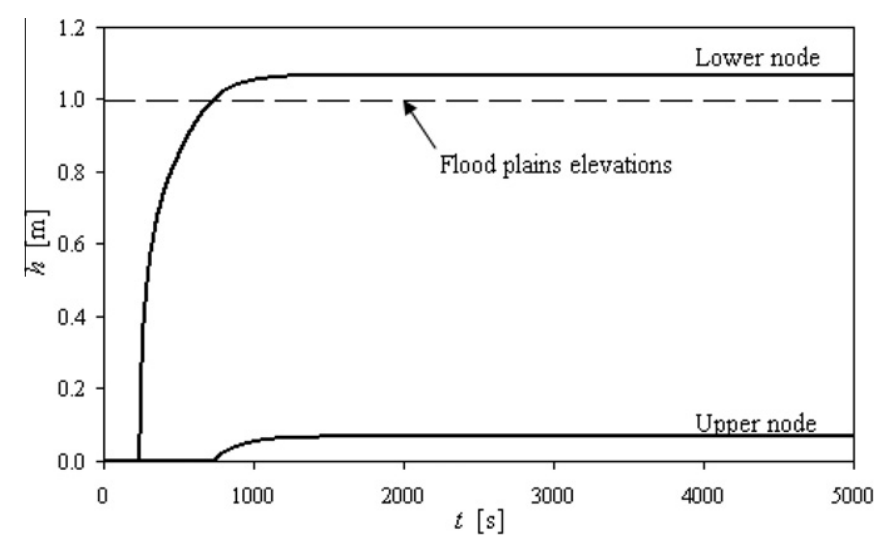

Fig. 31. Test 5 . Computed water depth at the monitored nodes.

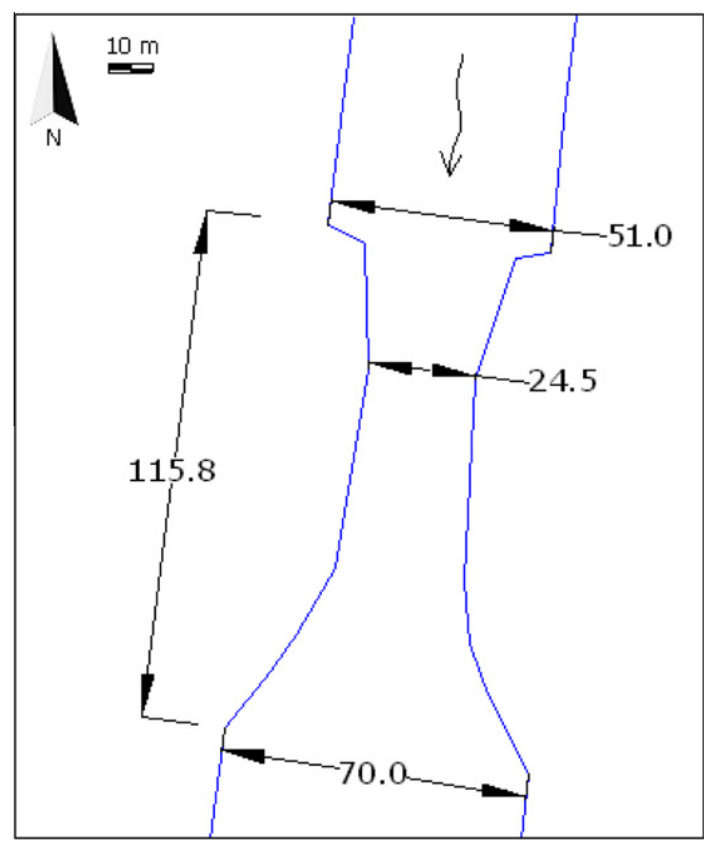

Fig. 32a. Test 6. Geometry of the Venturi channel.

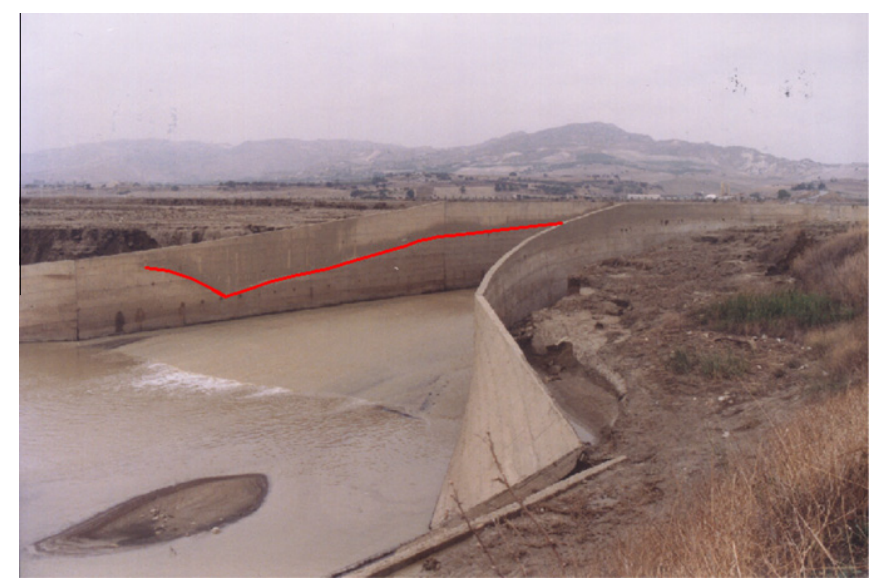

Fig. 32b. Test 6. Historical water surface profile. 


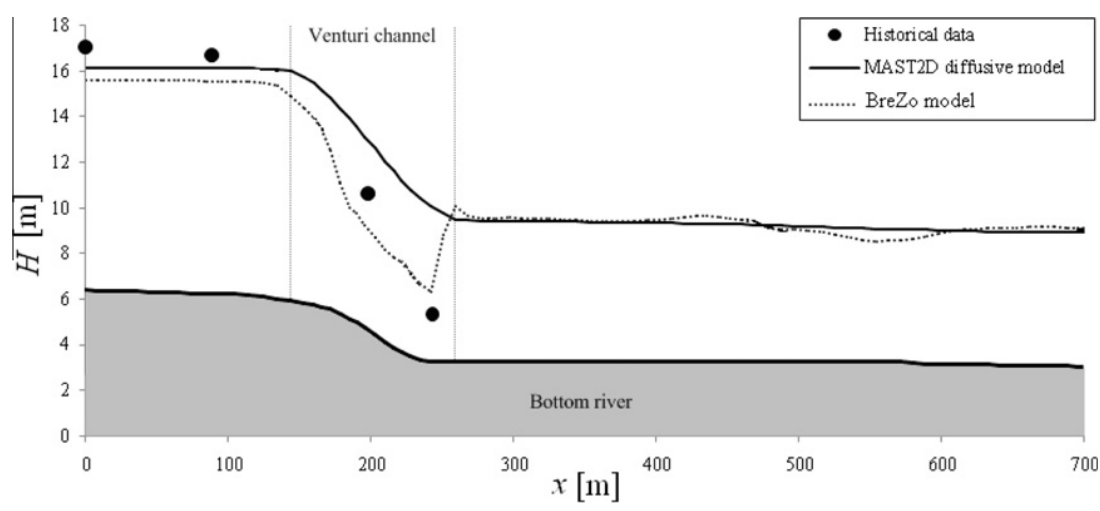

Fig. 33. Test 6. Steady-state profiles in the middle of the channel.

reached by the water surface during the event is marked with a red line.

In a previous study [36] the Manning's coefficient has been estimated equal to $0.04 \mathrm{~s} / \mathrm{m}^{1 / 3}$ and the maximum discharge, assumed constant along all the Venturi channel, equal to $1600 \mathrm{~m}^{3} / \mathrm{s}$. Normal flow depth has been assumed in the downstream section. In order to calculate the backwater profile, the roughness coefficient inside the restriction has been automatically calculated using the procedure described in Section 7.

The water levels computed by the proposed model have been compared with both the marked elevations and the steady-state water surface profile calculated by the complete model of BreZo using the same mesh.

Fig. 33 shows the central vertical cross section of the water surface profile. In spite of the many parameter uncertainties and errors, we can observe in the upstream section of the Venturi channel a good match between the results of the MAST model and the historical data, due to the reconstruction of the local energy dissipation carried out with the procedure explained in Section 7. Results of the complete model are more close to the historical data only inside the Venturi channel, where the diffusive model is unable to reproduce the profile of the supercritical flow.

\section{Conclusions}

A new algorithm for the simulation of parabolic 2D SW equations in strongly unstructured meshes has been developed, starting from the numerical structure of the previous DORA model [56]. The algorithm is aimed mainly to the simulation of gradually varying flows, with continuous head losses, but the backwater effect of two types of localized head losses, mainly the effect of vertical walls and hydraulic jumps, can be easily included in the model. The most important merit of the algorithm is the conservation of mass, the time step unconditional stability, its robustness with respect to abrupt parameter changes, like the topographic gradient, and, most important, the very low growth of the computational burden versus the number of nodes. The power law exponent of the computational burden is about 1.10 , that is very close to the value of the explicit algorithms.

The results of the proposed model have been compared with the results of a well-known complete model, with a completely different numerical structure. The results suggest that the use of diffusive models instead of a complete one can lead not only to computational time saving, but also to more accurate prediction, because of the smaller sensitivity of the diffusive model to the input topographic error with respect to the sensitivity of the complete model.
All these properties make the algorithm specially suited for its implementation in the context of early warning systems.

\section{Disclosure statement}

All the Authors of the paper, Costanza Aricò, Marco Sinagra, Lorenzo Begnudelli and Tullio Tucciarelli, disclose any actual or potential conflict of interest including any financial, personal or other relationships with other people or organizations within three (3) years of beginning the work submitted.

The Authors declare also that have participated in the research and article preparation and their individual contribution to the paper is equally distributed.

The Authors, Costanza Aricò, Marco Sinagra, Lorenzo Begnudelli and Tullio Tucciarelli Palermo, 11/04/2011.

\section{Acknowledgements}

The research has been funded by the PRIN 2008 program "Integration of hydraulic measurements in rivers for discharge and hydraulic resistance parameter monitoring".

\section{Appendix A. Mass conservation}

According to Eq. (16), it is possible to write Eq. (18a) as:

$\Delta t \overline{F l}_{i, j}^{\text {out }}=\left(\overline{F l}_{i}^{\text {in }} \Delta t-A_{i}\left(h_{i}^{k+1 / 2}-h_{i}^{k}\right)\right) \frac{K_{i, j}^{k}}{\Delta t \sum_{l} K_{i, l}^{k}}$

where

$\overline{F l_{i}^{i n}}=\bar{F} l_{i}^{i n}+A_{i} \overline{p_{i}}$

Summing all Eq. (A.1) for $i=1, \ldots, N$, one gets:

$\sum_{i} A_{i}\left(h_{i}^{k+1 / 2}-h_{i}^{k}\right)=\Delta t \sum_{i}\left(\overline{F l_{i}^{i n}}-\overline{F l_{i}^{\text {out }}}\right)$

Because for each linked couple of internal cells $i$ and $j$ (see Eq. $(14 b))$,

$\overline{F l}_{i, j}^{\text {out }}=\overline{F l}_{j, i}^{i n}$

the rhs of Eq. (A.3) is equal to the difference among the incoming and leaving boundary fluxes, plus the sum of the source terms applied to the computational domain (if different from zero), that is:

$\sum_{i} A_{i}\left(h_{i}^{k+1 / 2}-h_{i}^{k}\right)=\Delta t\left(F l_{\text {bou }}^{\text {in }}-F l_{\text {bou }}^{\text {out }}\right)+\widehat{P}$

where 
$\widehat{P}=\sum_{i} \overline{p_{i}} A_{i}$

and $F l_{\text {bou }}^{\text {in }}$ and $F l_{\text {bou }}^{\text {out }}$ are the sum of the incoming (assigned) and leaving convective boundary fluxes.

In the correction system (23), each term proportional to coefficient $D_{i, j}^{k}$ represents a diffusive flux between the linked cells $i$ and $j$. Diffusive fluxes are discretized as the convective ones and analogously to Eq. (A.4), the leaving diffusive flux from cell $i$ to cell $j$ is equal to the flux entering cell $j$ from cell $i$. The sum of all these terms, for $i=1, \ldots, N$, is zero, so that the sum of all Eq. (A.5) times $\Delta t$ gives:

$\sum_{i} A_{i}\left(h_{i}^{k+1}-h_{i}^{k+1 / 2}\right)=\Delta t F l_{d i r}$

where $F l_{\text {dir }}$ is the diffusive boundary flux computed through the Dirichlet nodes. Summing Eqs. (A.5)-(A.7) you finally get:

$\sum_{i} A_{i}\left(h_{i}^{k+1}-h_{i}^{k}\right)=\Delta t\left(F l_{\text {bou }}^{\text {in }}-F l_{\text {bou }}^{\text {out }}+F l_{\text {dir }}\right)+\widehat{P}$

that can be viewed as a global mass balance.

\section{Appendix B. Monotonicity of the steady-state solution for " $\mathrm{M}$ " systems}

The $M$-property implies that each diagonal element of the matrix $\mathbf{R}$ is opposite to the sum of all the extra-diagonal elements of the same equation. In steady-state condition the $i$ th linear equation can be written as:

$\sum_{j \neq i} H_{j} R_{i j}-H_{i} \sum_{j \neq i} R_{i j}=0$

as well as

$\sum_{j \neq i}\left(H_{j}-H_{i}\right) R_{i j}=0$

where all the extra-diagonal $R_{i j}$ coefficients are smaller than zero. The second equation implies that $H_{i}$ cannot be neither the minimum nor the maximum with respect to the surrounding $H_{j}$ values.

\section{Appendix C. Numerical flow vectors in the MAST procedure}

Assume a triangular element $m$ in the computational mesh, with nodes $i, j, l$ and assume for instance $H_{l}>H_{i}>H_{j}$. Fig. C.1 qualitatively shows the corresponding flow vector $\mathbf{q}=\mathbf{v} h$ according to the standard linear Galerkin FE scheme, where $\mathbf{v}$ is the averaged vertical velocity and $h$ is the water depth. In the same Galerkin FE scheme the flux given by Eq. (22b) is obtained by approximating the flow vector $\mathbf{q}$ in each triangle with a piecewise constant function, given by (see for example [30])

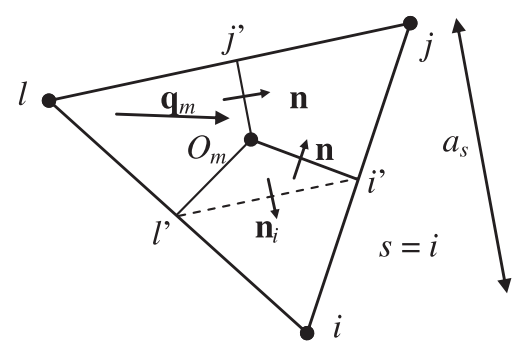

Fig. C.1. Velocity field in the standard conforming linear Galerkin FE scheme and notations. $\mathbf{q}_{m}=-\sum_{s=i, j, l} K^{m} \nabla w_{s}^{m} H_{s}$

where $K^{m}$ is the diffusion coefficient of element $m$ that, with the notation used in this paper (see Eq. (22b)), is:

$K^{m}=E_{m}^{k} \hat{h}_{m}^{5 / 3}$

where $w_{s}^{m}$ is the piecewise linear Galerkin basis functions for node $s$ in the element $m$ and $\nabla w_{s}^{m}$ is its constant gradient. $w_{s}^{m}$ is a linear function, equal to one on node $s$ and zero on the other nodes. $\nabla w_{s}^{m}$ is a constant vector orthogonal to side opposite to node $s$, with module $1 / a_{s}\left(a_{s}\right.$ is the triangle height measured from node $s$ to the opposite side (see Fig. C.1)), that can be written as [43] (see Fig. C.1):

$\nabla w_{s}^{m}=\frac{\mathbf{n}_{s}}{a_{s}}$

where $\mathbf{n}_{s}$ is the unitary vector pointing to node $s$, orthogonal to the opposite side.

Fluxes between cells $i$ and $j$ and between cells $i$ and $l$, given in Eq. (22b), are:

$F l_{i, j}^{o u t, m}=d_{i, j}^{m} E_{m}^{k} \frac{H_{i}^{k}-H_{j}^{k}}{d_{i j}} \hat{h}_{m}^{5 / 3}=K^{m} d_{i, j}^{m} \frac{H_{i}^{k}-H_{j}^{k}}{d_{i j}}$

$F l_{i, l}^{\text {out }, m}=d_{i, l}^{m} E_{m}^{k} \frac{H_{l}^{k}-H_{i}^{k}}{d_{i l}} \hat{h}_{m}^{5 / 3}=K^{m} d_{i, l}^{m} \frac{H_{l}^{k}-H_{i}^{k}}{d_{i l}}$

Fluxes through the segment $\overline{i^{\prime} O_{m}}$ and $\overline{l^{\prime} O_{m}}$ connecting midpoint $i^{\prime}$ of side $i j$ and midpoint $l^{\prime}$ of side $i l$ with the triangle circumcentre $O_{m}$ (see Fig. C.1), due to a unitary piezometric heads difference between cells $i$ and $j$ and between cells $i$ and $l$ are respectively equal to [43]:

$b_{i j}^{m}=\int_{\varsigma_{i j}}-K^{m} \nabla w_{i}^{m} \cdot d \mathbf{n}$

$b_{i l}^{m}=\int_{\varsigma_{i l}}-K^{m} \nabla w_{i}^{m} \cdot d \mathbf{n}$

where $\varsigma_{i j}=\overline{i^{\prime} O_{m}}, \varsigma_{i l}=\overline{l^{\prime} O_{m}}$ and $\mathbf{n}$ is the unitary normal to $\varsigma_{i j}\left(\varsigma_{i l}\right)$ pointing opposite to node $i$. The sum of the fluxes between cells $i$ and $j$ and between cells $i$ and $l$ in element $m$ is:

$$
\begin{aligned}
\sum_{s \neq i}\left(H_{s}^{k}-H_{i}^{k}\right)\left(\int_{\varsigma_{i s}}-K^{m} \nabla w_{i}^{m} \cdot d \mathbf{n}\right) & =\sum_{s} b_{i s}^{m} H_{s}^{k} \\
& =b_{i j}^{m} H_{j}^{k}+b_{i l}^{m} H_{l}^{k}+b_{i i}^{m} H_{i}^{k}
\end{aligned}
$$

where $s=j, l$ and $b_{i i}^{m}=-b_{i j}^{m}-b_{i l}^{m}$. According to Eqs. (C.1) and (C.2) can be written as:

$b_{i j}^{m} H_{j}^{k}+b_{i l}^{m} H_{l}^{k}+b_{i i}^{m} H_{i}^{k}=\sum_{s \neq i} \int_{\varsigma_{i s}} \mathbf{q}_{m} \cdot d \mathbf{n}$

Eq. (C.7) represents the sum of the fluxes between cells $i$ and $j$ and between cells $i$ and 1 given by Eqs. (C.4), that are the fluxes through the boundary of the Voronoi cell of node $i$ lying in element $m$. Applying this procedure to all triangles sharing node $i$, the flux through the boundary of the Voronoi cell of node $i$ is obtained.

The same steps can be followed in the MAST approach to obtain the fluxes computed in Eq. (22a), that are:

$$
\begin{aligned}
& F l_{i, j}^{\text {out }, m}=c_{i, j}^{m} E_{m}^{k} \frac{H_{i}^{k}-H_{j}^{k}}{d_{i j}} h_{s}^{5 / 3}=K^{\prime m} c_{i, j}^{m} \frac{H_{i}^{k}-H_{j}^{k}}{d_{i j}}, \quad K^{\prime m}=E_{m}^{k} h_{s}^{5 / 3} \\
& \text { with } s=i \text { if } H_{i}^{k} \geqslant H_{j}^{k}, s=j \text { if } H_{i}^{k}<H_{j}^{k} \text { and } \\
& F l_{i, l}^{\text {out }, m}=c_{i, l}^{m} E_{m}^{k} \frac{H_{l}^{k}-H_{i}^{k}}{d_{i l}} h_{s}^{5 / 3}=K^{\prime m} c_{i, l}^{m} \frac{H_{l}^{k}-H_{i}^{k}}{d_{i l}}, \quad K^{\prime m}=E_{m}^{k} h_{s}^{5 / 3}
\end{aligned}
$$




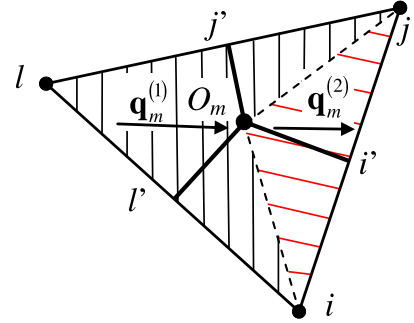

Fig. C.2. Velocity field in the MAST scheme.

with $s=i$ if $H_{i}^{k} \geqslant H_{l}^{k}, s=l$ if $H_{i}^{k}<H_{l}^{k}$. To get the flux through the edges of the Voronoi cells given by Eq. (22a), two different flow vectors have to be assigned in two different parts of each element. According to the above assumption $H_{l}>H_{i}>H_{j}$, the two flow vectors are:

$\mathbf{q}_{m}^{(1)}=-\sum_{s=i, j, l} E_{m}^{k} h_{l}^{5 / 3} \nabla w_{s}^{m} H_{s}$
$\mathbf{q}_{m}^{(2)}=-\sum_{s=i, j, l} E_{m}^{k} h_{i}^{5 / 3} \nabla w_{s}^{m} H_{s}$

See in Fig. C. 2 a possible partition of element $m$. All the partitions that leave the edges of the Voronoi cell of node $l$ inside the sub-element with flow vector $\mathbf{q}_{m}^{(1)}$ and the remaining edge of Voronoi cell of node $i$ in the sub-element with flow vector $\mathbf{q}_{m}^{(2)}$ provide the same fluxes given by Eq. (22a).

Observe that in both standard Galerkin FE scheme as well as MAST scheme flow vectors have discontinuous normal components across sub-element boundary. This leads the velocity field satisfies the mass balance equation only in the weak sense [44].

\section{Appendix D. Computation of a generalized Delaunay mesh}

In a given triangulation, call $e_{1}$ and $e_{2}$ two triangles with nodes $i$ $j, k$ and $i, j, m$, sharing edge $i j$. The two triangles will satisfy the Delaunay condition with respect to edge $i j$ if:

(1) the distances $d_{i, j}^{k, 1}$ and $d_{i, j}^{m, 2}$ of both nodes $k$ and $m$ with respect to the midpoint of edge $i j$ are greater than $d_{i j} / 2$, where $d_{i j}$ is the distance between nodes $i$ and $j$.

The proof of the previous statement is the following. Observe that according to condition (1) nodes $k$ and $m$ will be external to the circle having the centre on the midpoint of edge $i j$ and radius $d_{i j} / 2$. All the points on this circle form with nodes $i$ and $j$ right triangles, all the external nodes acute triangles, all the internal nodes obtuse triangles. Because $d_{i, j}^{k, 1}>d_{i j} / 2$ and $d_{i, j}^{m, 2}>d_{i j} / 2$, both triangles with nodes $i, j, k$ and $i, j, m$ are acute triangles and edge $i j$ satisfies the Delaunay property.

For a similar reason, the distance of the circumcentre of each triangle with nodes $i, j, k$ from its edge $i j$ on the external boundary is always positive if:

(2) the distance $d_{i, j}^{k, 1}$ of node $k$ with respect to the midpoint of edge $i j$ is greater than $d_{i j} / 2$.

To guarantee the convergence of the swap iterative process to a mesh that satisfies conditions (25), without changing the edges that are overlapping internal boundaries, it will be sufficient to: (a) start with a mesh that has all the internal and external boundaries overlapping one or more element edges $i j$ and (b) guarantee that condition (1) (or (2)) is satisfied for all the potential triangles formed by edges $i j$ and a third node. This is true if all the nodes in the mesh different from $i$ and $j$ have a distance from the midpoint of $i j$ greater than $d_{i j} / 2$ and this can be easily obtained by reducing the length of the edges $i j$.

\section{Appendix E. Matrix M-property of the proposed correction system}

The linear system (23a) can be written in vector-matrix form as:

$\mathbf{R} \boldsymbol{\eta}=\mathbf{b}$

where

$R_{i i}=\frac{A_{i}}{\Delta t}+\sum_{n=1, N_{T}} D_{i, j}^{k} \delta_{i, n}$

$R_{i j}=-\sum_{n=1, N_{T}} D_{i, j}^{k} \delta_{i, n} \quad$ for $i \neq j$

and

$b_{i}=\sum_{n=1, N_{T}} D_{i, j}^{k}\left(\vartheta_{j}-\vartheta_{i}\right) \delta_{i, n}$

Because all coefficients $D_{i, j}^{k}$ computed by Eq. (23b) are positive, all the extra-diagonal coefficients (E.3) are negative and all diagonal coefficients (E.2) are positive and greater or equal than the opposite of the sum of all the extra-diagonal coefficients of the same equation.

\section{References}

[1] Aizinger V, Dawson CN. Discontinuous Galerkin methods for two-dimensional flow and transport in shallow water. Adv Water Res 2002;25:67-84.

[2] Alcrudo F, Garcia-Navarro P. A high-resolution Godunov-type scheme in finite volumes for the 2D shallow water equations. Int J Numer Methods Fluids 1993; 16:489-505.

[3] Alcrudo F, Benkhaldoun F. Exact solution to the Riemann problem of the shallow-water equations with a bottom step. Comput Fluids 2001:30:643-71.

[4] Anastasiou K, Chan CT. Solution of the 2D shallow water equations using the finite volume method on unstructured triangular meshes. Int J Numer Methods Fluids 1997;24:1225-45.

[5] Aricò C, Tucciarelli T. MAST solution of advection problems in irrotational flow fields. Adv Water Res 2007;30(3):665-85.

[6] Aricò C, Tucciarelli T. A Marching in space and time (MAST) solver of the shallow water equations. Part I: The 1D case. Adv Water Res 2007;30(5): 1236-52.

[7] Aricò C, Tucciarelli T. The MAST FV/FE scheme for the simulation of twodimensional thermohaline processes in variable-density saturated porous media. J Comput Phys 2009;228(4):1234-74.

[8] Aricò C, Nasello C, Tucciarelli T. A Marching in space and time (MAST) solver of the shallow water equations. Part II: The 2D case. Adv Water Res 2007;30(5): 1253-71.

[9] Balzano A. Evaluation of methods for numerical simulation of wetting and drying in shallow water flow models. Coastal Eng 1998;34:83-107.

[10] Bascià A, Tucciarelli T. An explicit unconditionally stable numerical solution of the advection problem in irrotational flow fields. Water Resour Res 2004;40(6):W06501.

[11] Bates PD, De Roo APJ. A simple raster-based model for flood inundation simulation. J Hydrol 2000;236:54-77.

[12] Begnudelli L, Sanders BF. Conservative wetting and drying methodology for quadrilateral grid finite volume models. J Hydraul Eng, ASCE 2007:133(3): 312-22.

[13] Begnudelli L, Sanders BF. Unstructured grid finite-volume algorithm for shallow-water flow and scalar transport with wetting and drying. J Hydrau Eng, ASCE 2006;132(4):371-84.

[14] Bermudez A, Vàzquez ME. Upwind methods for hyperbolic conservation laws with source terms. Comput Fluids 1994;8:1049-71.

[15] Chippada S, Dawson CN, Martinez M, Wheeler M. A Godunov-type finite volume method for the system of shallow water equations. Comput Methods Appl Mech Eng 1998;151:105-29.

[16] Cunge J, Holly FM, Verwey A. Practical aspects of computational river Hydraulics. London: Pitman ed., Advanced Publishing Program; 1980.

[17] Daluz Vieira JH. Conditions governing the use of approximations for the SaintVenant equations for shallow water flow. J Hydrol 1983;60:43-58.

[18] Dawson CN, Proft J. Discontinuous and coupled continuous/discontinuous Galerkin methods for the shallow water equations. Comput Methods Appl Mech Eng 2002;191:4721-46. 
[19] Dooge JCI, Harley BM. Linear routing in uniform channels. In: Proceedings of the international hydrology symposium. Fort Collins, CO: Colorado State University; 1967.

[20] Forsyth PA. A control volume finite element approach to NAPL groundwater contamination. SIAM J Sci Statist Comput 1991;12:1029-57.

[21] Gourgue O, Comblen R, Lambrechts J, Kärnä T, Legat V, Deleersnijder E. A fluxlimiting wetting-drying method for finite-element shallow-water models, with application to the Scheldt Estuary. Adv Water Res 2009;32:1726-39.

[22] Guinot V, Cappeleare B. Sensitivity analysis of 2D steady-state shallow water flow. Application to free surface model calibration. Adv Water Res 2009;32: 540-60.

[23] Horrit MS, Bates PD. Evaluation of 1D and 2D numerical models for predicting river flood inundation. J Hydrol 2002;268:87-99.

[24] Hunter NM, Horrit MS, Bates PD, Wilson MD, Werner MGF. An adaptive time step solution for raster-based storage cell modelling of floodplain inundation. Adv Water Res 2005;28:975-91.

[25] Huthoff F, Roos PC, Augustijn DCM, Hulscher S. Interacting divided channel method for compound channel flow. J Hydraul Eng, ASCE 2008;134(8): 1158-65.

[26] Kärnä T, de Brye B, Gourgue O, Lambrechts J, Comblen R, Legat V, et al. A fully implicit wetting-drying method for DG-FEM shallow water models, with an application to the Scheldt Estuary. Comput Methods Appl Mech Eng 2011;200(5-8):509-24.

[27] Kawahara M, Hirano H, Tsuhora K, Iwagaki K. Selective lumping finite element method for shallow water equations. Int J Numer Methods Eng 1982;2: 99-112.

[28] King I, Norton WR. Recent application of RMAs finite element models for twodimensional hydrodynamics and water quality. In: Brebbia C, Gray WG, Pinder GF, editors. Finite elements in water resources, vol. II. London: Pentech Press; 1978.

[29] Klausen RA, Russell TF. Relationships among some locally conservative discretization methods which handle discontinuous coefficients. Comput Geosci 2004;8(4):341-77.

[30] Lapidus L, Pinder GF. Numerical solution of partial differential equations in science and engineering. New York: John Wiley; 1982.

[31] LeVeque RJ. Balancing source terms and flux gradients in high-resolution Godunov methods: the quasi-steady wave-propagation algorithm. J Comput Phys 1998;146(1):346-65.

[32] Liand Q, Borthwick AGL, Stelling G. Simulation of dam and dyke-break hydrodynamics on dynamically adaptive quadtree grids. Int J Numer Methods Fluids 2004;46:127-62.

[33] Lynch DR, Gray WR. A wave equation model for finite element computations. Comput Fluids 1979;7:207-28.

[34] Mingham CG, Causon DM. A high resolution finite volume method for the shallow water equations. J Hydraul Eng, ASCE 1998;124(6):605-14.

[35] Moussa R, Boucquillon C. On the use of the diffusive wave for modelling extreme flood events with overbank flow in the floodplain. J Hydrol 2009;374:116-35

[36] Nasello C, Aronica G, Tucciarelli T. Un modello bidimensionale per la propagazione delle piene in aree urbane ed extraurbane. Atti del XXVII Convegno di Idraulica e Costruzioni Idrauliche, vol. III, Genova, settembre 2000. p. 77-83 [in Italian].

[37] NETGEN http://sourceforge.net/projects/netgen-mesher.

[38] Noto V, Tucciarelli T. DORA Algorithm for network flow models with improved stability and convergence properties. J Hydraul Eng, ASCE 2001;127(5): 380-91.
[39] Ponce VM, Li RM, Simons DB. Applicability of kinematic and diffusion models. J Hydraul Div, ASCE 1978;104:353-60.

[40] Ponce VM. Engineering hydrology, principles and practices. Englewood Cliffs, NJ: Prentice Hall; 1989.

[41] Ponce VM. Generalized diffusive wave equation with inertial effects. Water Resour Res 1990;26:1099-101.

[42] Putti M, Cordes C. Finite element approximation of the diffusion operator on tetrahedral. SIAM J Sci Comput 1998;19(4):1154-68.

[43] Putti M, Sartoretto F. Linear Galerkin vs mixed finite element 2D flow fields. Int J Numer Methods Fluids 2009;60:1011-31.

[44] Quarteroni A, Valli A. Numerical approximations of partial differential equations. Springer series in computational mathematics, vol. 23. Berlin: Springer; 1994.

[45] Saint-Venant B. Théorie du mouvement non permanent des eaux, avec application aux crues des rivières et à l'introduction des marées dans leurs lits. Comptes Rendus des Séances de l'Académie des Sciences 73, 1871. p. 14754, 237-40.

[46] Schöberl J. NETGEN - An advancing front 2D/3D-mesh generator based on abstract rules. Comput Visualiz Sci 1997;1(1):41-52.

[47] Singh VP. Accuracy of kinematic wave and diffusion wave approximations for space independent flows. Hydrol Process 1994;8:45-62.

[48] Singh VP. Accuracy of kinematic wave and diffusion wave approximations for time independent flows. Hydrol Process 1995;9:755-82.

[49] Singh VP. Kinematic wave modelling in water resources: surface water hydrology. New York: John Wiley \& Sons Ltd.; 1996.

[50] Smith MB, Seo DJ, Koren VI, Reed SM, Zhang Z, Duan Q, et al. The distributed model intercomparison project (DMIP): motivation and experiment design. J Hydrol 2004;298:4-26.

[51] Subramanya K. Flow in open channels, 3rd ed. Edt. Tata McGraw-Hill; 2009.

[52] Szymkiewicz R. Oscillation-free solution of shallow water equations for nonstaggered grid. J Hydraul Eng, ASCE 1993;119:1118-37.

[53] Tang X-N, Knight DW, Samuels PG. Volume conservation in variable parameter Muskingum-Cunge method. J Hydraul Eng, ASCE 1999;125(6):610-20.

[54] Toro EF. shock-capturing methods for free-surface shallow flows. Chichester: John Wiley \& Sons; 2001.

[55] Tsai WC. Applicability of kinematic, noninertia and quasy-steady dynamic wave model to unsteady flow routing. J Hydraul Eng, ASCE 2003;129(8): 613-27.

[56] Tucciarelli T, Termini D. Finite-element modelling of floodplain flows. J Hydraul Eng, ASCE 2000;126(6):416-24.

[57] Vazquez-Cendon ME. Improved treatment of source terms in upwind schemes for the shallow water equations in channels with irregular geometry. J Comput Phys 1999;148:497-526.

[58] Weinmann PE, Laurenson EM. Approximate flood routing methods: a review. J Hydraul Div, ASCE 1974;105:1521-36.

[59] Younes A, Ackerer P, Lehmann F. A new mass lumping for the mixed hybrid finite element method. Int J Numer Methods Eng 2006;67:89-107.

[60] Yu D, Lane SN. Urban fluvial flood modelling using a two-dimensional diffusion-wave treatment, part 1: mesh resolution effects. Hydrol Process 2006;20:1541-65.

[61] Zhou JG, Causon DM, Ingram DM, Mingham CG. Numerical solution of the shallow water equations with discontinuous bed topography. Int J Numer Methods Fluids 2002;38:769-88.

[62] Zienkiewicz O, Ortiz P. A split-characteristic based finite element model for the shallow water equations. Int J Numer Methods Fluids 1995;20:1061-80. 